\title{
1 Aging-regulated TUG1 is dispensable for endothelial cell
}

\section{2 function}

Anna Theresa Gimbel'1,2, Susanne Koziarek¹, Kosta Theodorou ${ }^{1}$, Jana Felicitas $\mathrm{Jo}^{7,8}$, Norbert Hübner ${ }^{3,4,9}$, Lars Maegdefessel ${ }^{10,11,12}$, Stefanie Dimmeler ${ }^{1,2}$, Sebastiaan van Heesch ${ }^{13}$ and Reinier A. Boon ${ }^{1,2,5},{ }^{*}$

${ }^{1}$ Centre of Molecular Medicine, Institute of Cardiovascular Regeneration, Goethe-University, Frankfurt am Main, Germany. Helmholtz Association (MDC), Berlin, Germany.

5Department of Physiology, Amsterdam Cardiovascular Sciences, VU University Medical Centre, Amsterdam, Netherlands.

6Max Planck Institute for Heart and Lung Research, Bioinformatics and Deep Sequencing Platform, Bad Nauheim, Germany.

${ }^{7}$ Wallace H. Coulter Department of Biomedical Engineering, Georgia Institute of Technology and Emory University, Atlanta, United States.

${ }^{8}$ Division of Cardiology, Emory University, Atlanta, United States.

${ }^{9}$ Charité-Universitätsmedizin, Berlin, Germany.

${ }^{10}$ Department of Vascular and Endovascular Surgery, Technical University Munich, Munich, Germany.

${ }^{11}$ German Center for Cardiovascular Research DZHK, Partner Site Munich, Germany.

${ }^{12}$ Department of Medicine, Karolinska Institute, Stockholm, Sweden.

${ }^{13}$ Princess Máxima Center for Pediatric Oncology, Utrecht, The Netherlands.

\footnotetext{
* Corresponding author: r.a.boon@amsterdamumc.nl (RAB)
} 


\section{Abstract}

37 The evolutionary conserved Taurine Upregulated Gene 1 (TUG1) is a ubiquitously expressed gene that is one of the highest expressed genes in human and rodent endothelial cells (ECs). We here show that TUG1 expression decreases significantly in aging mouse carotid artery ECs and human ECs in vitro, indicating a potential role in the aging endothelial vasculature system. We therefore investigated if, and how, TUG1 might

42 function in aging ECs, but despite extensive phenotyping found no alterations in basal EC proliferation, apoptosis, barrier function, migration, mitochondrial function, or monocyte adhesion upon TUG1 silencing in vitro. TUG1 knockdown did slightly and significantly decrease cumulative sprout length upon vascular endothelial growth factor A stimulation in human umbilical vein endothelial cells (HUVECs), though TUG1-silenced HUVECs

47 displayed no transcriptome-wide mRNA expression changes explaining this effect.

48 Further, ectopic expression of the highly conserved and recently discovered 153 amino

49 acid protein translated from certain TUG1 transcript isoforms did not alter angiogenic sprouting in vitro. Our data show that, despite a high expression and strong evolutionary

51 conservation of both the TUG1 locus and the protein sequence it encodes, TUG1 does 52 not seem to play a major role in basic endothelial cell function. 


\section{Introduction}

55 In the last decade, non-coding RNAs, and especially long non-coding RNAs (IncRNAs), 56 were implicated in the development of aging-induced cardiovascular diseases (CVDs) (1-

57 4). Taurine upregulated gene 1 (TUG1), a well-studied IncRNA in many types of cancer

58 (5), was previously associated with diabetic retinopathy in mice being an aging-induced disease (6-8). Furthermore, TUG1 has been described to be involved in tumor-induced angiogenesis $(9,10)$. However, the role of TUG1 in aging-induced CVDs remains largely

61 unknown.

63 CVDs are the leading cause of death worldwide (11). Each year CVDs cause over 1.8 64 million deaths in the European Union including myocardial infarction, stroke, atrial 65 fibrillation, vascular diseases and many more (12). A prominent risk factor for the development of CVDs is aging. The increasing number of the elderly is already a great

67 challenge for the health care system that needs to be engaged within the next years. On 68 top of that, the population over 65 years is estimated to double from $12 \%$ in 2010 to $22 \%$ 69 in $2040(13)$

71 At structural level, aging correlates with several changes in the vasculature: It leads to 72 stiffening of the vessel wall, thickening of the intima, endothelial dysfunction and increased

73 vascular inflammation (14). Additionally, aging leads to impaired angiogenesis, and a 74 diminished angiogenic response to injuries, both important mechanisms in the 75 development of CVDs (15). Angiogenesis describes the outgrowth of new vessels from 
pre-existing ones via a cascade of highly coordinated cellular functions driven by pro-

77 angiogenic stimuli (16). Embryonic development, patterning of the vascular system and wound healing rely on the precise coordination of migrating and quiescent endothelial cells (17). Conversely, pathological angiogenesis is involved in malignant, inflammatory, immune and ischemic disorders $(16,18)$.

82 LncRNAs are commonly characterized to be more than 200 nucleotides long, poorly conserved among species and expressed in a tissue-specific manner (19). Via control of epigenetic $(20,21)$, transcriptional $(22,23)$ and post-transcriptional processes $(24,25)$, IncRNAs regulate various biological functions. TUG1 is, in contrast to other well-studied IncRNAs, highly conserved among different species and ubiquitously expressed with moderate to high expression in different adult tissues in human and mouse $(26,27)$.

TUG1 was initially identified as a crucial IncRNA in the development of photoreceptors in the mouse retina (28). In cancer, TUG1 acts in a tissue- or context-specific manner either

91 as tumor-suppressor or oncogene by affecting cancer cell proliferation, migration and 92 invasion (28-30, 27, 5, 21). Mechanistically, TUG1 can recruit Polycomb-repressive complex 2 (PRC2) and repress the expression of specific target genes in trans in the nucleus (30). Furthermore, TUG1 can sequester micro RNAs (miR) in the cytoplasm, e.g. miR-145 in gliomas, further implementing a role in (epi-) transcriptomic regulation (21).

98 The general expectation that non-coding RNAs do not exhibit any coding potential is 
analyses propose the translation of a larger portion of the genome than previously accepted (31). Instead, it is believed that about $22 \%$ of the transcribed IncRNAs are translated into microproteins (26). Representative IncRNAs that were identified to show translation, are Long Intergenic Non-Protein Coding RNA, P53 Induced Transcript (LINCPINT), Differentiation Antagonizing Non-Protein Coding RNA (DANCR), Plasmacytoma Variant Translocation 1 (PVT1) and many more (26). In this study, van Heesch et al. identified an open reading frame (ORF) in a previously misannotated 5'-leader sequence of the TUG1 transcript starting with the non-canonical start-codon CUG. Translation of the TUG1 protein (153 amino acids) was demonstrated via sequence conservation analyses, ribosome profiling, coupled in vitro transcription: translation assays, and ectopic expression of tagged constructs followed by Western blot and immunofluorescence microscopy. Functionally, the TUG1 protein has been described to localize to mitochondria and influence mitochondrial bioenergetics $(27,26)$. These various lines of coding-sequence evidence, together with the high amino acid sequence conservation of the TUG1 ORF across species, over the course of this project led to TUG1's official classification as a protein-coding gene (Ensembl release v100; April 2020).

The influence of TUG1 on mitochondrial bioenergetics is further emphasized in the context

118 of diabetic retinopathy. Murine Tug1 positively regulates Ppargc1a gene transcription and

119 its target genes in podocytes in mice by acting as a scaffold between an enhancer

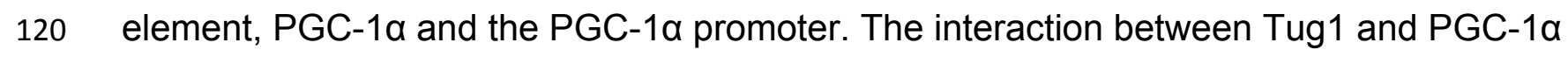
121 increased mitochondrial content, mitochondrial respiration and cellular ATP levels and 122 reduced mitochondrial ROS (6). Male Tug1 knockout mice are sterile with underlying 
123 defects including a low number of sperm and an abnormal sperm morphology originating

124 from impaired spermatogenesis (27).

126 Here, we characterized the IncRNA TUG1 and its role in endothelial cell function. We 127 showed that TUG1 is regulated by aging in endothelial cells in vitro and in vivo. TUG1

128 silencing did not change basal endothelial function addressing proliferation, apoptosis, 129 migration, barrier function, mitochondrial function or monocyte adhesion. We identified a 130 small impact of TUG1 silencing on VEGFA-stimulated angiogenic sprouting in vitro. TUG1 131 does not influence transcription supporting a dispensability in ECs. TUG1 proteins were 132 expressed without having any effects on VEGFA-stimulated sprouting. 


\section{Methods}

\section{Cell culture}

HUVECs (pooled donor; Lonza) were cultured in endothelial basal medium (EBM; Lonza)

137 supplemented with 10\% fetal calf serum (FCS; Invitrogen) and EGM-SingleQuots (Lonza).

138 Cells were cultured at $37^{\circ} \mathrm{C}$ with $5 \% \mathrm{CO}_{2}$. For the different assays passages 2 or 3 of

139 HUVECs were used. Human embryonic kidney cells (Hek293T; DSMZ; \# ACC 16) (32)

140 were cultured in DMEM with $10 \%$ heat-inactivated FCS, D-glucose, pyruvate and

141 Penicillin/streptomycin. Cells were cultured at $37^{\circ} \mathrm{C}$ and $5 \% \mathrm{CO}_{2}$. Cell numbers were 142 determined with the Nucleocounter NC-2000 (Chemometec A/S).

\section{Transfection and lentiviral overexpression}

145 HUVECs were transfected at $60 \%$ confluency with $10 \mathrm{nmol} / \mathrm{L}$ locked nucleic acid (LNA) 146 GapmeRs (Qiagen) or small interfering RNAs (siRNAs; Sigma Aldrich) using

147 Lipofectamine RNAiMAX (Life Technologies) according to the manufacturer's protocol. A 148 siRNA against firefly luciferase (Sigma Aldrich) or scrambled LNA GapmeR (Qiagen, 339516) were transfected as controls. The medium was changed to EBM (Lonza)

150 supplemented with 10\% FCS (Invitrogen) and EGM SingleQuots (Lonza) after 4h.

151 GapmeR and siRNA sequences are listed in the Supplement.

152 Lentivirus stocks were produced in Hek293T cells using pCMVDR8.91 as packaging 153 plasmid and pMD2.G (Addgene \#12259) as vesicular stomatitis virus G glycoprotein

154 (VSV-G) envelope expressing plasmid (33). In brief, 10x106 Hek293T were seeded 24h 155 before transfection. Cells were transfected with $10 \mu g$ pLenti4-V5 plasmid, $6 \mu$ g packaging 7 
plasmid pCMV $\Delta$ R8.91 and $2 \mu \mathrm{g}$ VSV-G plasmid pMD2.G. Empty pLenti4-V5 vectors were

used as control (mock). HUVECs were transduced for $24 \mathrm{~h}$. Medium was changed daily

Virus was concentrated by usage of LentiX Concentrator (TakaraBio) according to the condition and stored at $-80^{\circ} \mathrm{C}$.

One day before transfection, HUVECs were seeded in a density of $3 \times 10^{5}$ cells. HUVECs

were transduced with one aliquot of virus. Cells were washed $24 \mathrm{~h}$ and $72 \mathrm{~h}$ after transduction 3 times in an alternating order with PBS and EBM.

167 Plasmid cloning

168 For the overexpression of the TUG1 ORF, three different constructs with a C-terminal

FLAG-tag were subcloned from pEF1a vectors (26) into pLenti4-V5 backbones: pLenti4and downstream sequences) as a template and the addition of the TOPO site in parallel.

173 The pENTR ${ }^{\text {TM} / D-T O P O ® ~ C l o n i n g ~ K i t ~(I n v i t r o g e n) ~ u t i l i z e s ~ a ~ h i g h l y ~ e f f i c i e n t ~ " T O P O ~ C l o n i n g " ~}$

174 strategy to directionally clone the blunt-end PCR product into the pENTR vector as an 175 entry point into the Gateway ${ }^{\circledR}$ System. Identification of positive transformants was 176 achieved by transformation of One Shot ${ }^{\mathrm{TM}}$ Stbl3 ${ }^{\mathrm{TM}}$ Chemically Competent E. coli 177 (Invitrogen) and subsequent analysis by restriction analysis with EcoRV-HF (New England 178 Biolabs) and sequencing. Gateway® LR Clonase $®$ II enzyme mix (Invitrogen) catalyzed 179 the in vitro recombination between and the entry clone pENTR (containing the gene of 8 
interest flanked by attL sites) and the pLenti4-V5 destination vector (containing attR sites)

181 to generate an expression clone by homologous recombination. The analysis of the resulting clones was performed by restriction analysis with Ncol-HF (New England Biolabs), for the exclusion of unwanted recombination events, and sequencing.

\section{Western blot}

HUVECs were lysed with Radioimmunoprecipitaion assay (RIPA) buffer (Thermo Fisher) supplemented with Halt ${ }^{\mathrm{TM}}$ Protease and Phosphatase Inhibitor Cocktail (1:100; Thermo debris was removed by centrifugation $\left(16000 \mathrm{xg}\right.$ for $10 \mathrm{~min}$ at $\left.4^{\circ} \mathrm{C}\right)$. Protein concentration was determined by Pierce ${ }^{\mathrm{TM}}$ BCA Protein Assay Kit (Thermo Fisher Scientific). Equal amounts of denaturated protein in Laemmli buffer were loaded on $12 \%$ Sodium dodecyl sulfate gels (BioRad) and blotted on nitrocellulose membranes (Invitrogen). Membranes were blocked with $3 \%$ milk (Roth) in TBS-T and incubated with primary antibody overnight at $4^{\circ} \mathrm{C}$ under rotation. Secondary antibodies tagged with Horse Radish Peroxidase (HRP; using enhanced chemiluminescence (ECL, Thermo Fisher) on the ChemiDoc device (BioRad). Band intensity was quantified using ImageLab Software (version 5.2.1; BioRad). Antibodies and dilutions can be found in S1 Table.

\section{RT-qPCR}

201 Total RNA was isolated from HUVECs with the RNA Direct-zol RNA miniprep Kit 202 (Zymoresearch) by following the manufacturer's protocol including DNase digest. For 
using random hexamer primers (Thermo Fisher) and Multiscribe reverse transcriptase

(Applied Biosystems). The resulting copy DNA (cDNA) was used as template for RT-

qPCR in combination with Fast SYBR Green Master Mix (Applied Biosystems) in an

Applied Biosystems StepOnePlus machine (Applied Biosystems) or Viia7 device (Applied

Biosystems). Human ribosomal protein (RPLP0), glyceraldehyde-3-phosphate (GAPDH)

or TATA-Box Binding Protein (TBP) were used for normalization. Gene expression

211 Table.

\section{RNA Sequencing}

HUVECs were transfected with Control or TUG1 GapmeRs. Total RNA was isolated after

$48 \mathrm{~h}$ with Qlazol (Qiagen) and the Direct-zol RNA miniprep kit (Zymo Research) according

to the manufacturer's instructions including DNase digest. Alternatively, total RNA was

isolated from cell pellets from cardiomyocytes, aortic fibroblasts, pericytes, aortic smooth endothelial cells, saphenous vein endothelial cells, pulmonary microvascular endothelial cells, dermal microvascular endothelial cells, cardiac microvascular endothelial cells, coronary artery endothelial cells, pulmonary artery endothelial cells and aortic endothelial

222 cells (all human; Promocell) with the miRNeasy Micro Kit (Qiagen) according to the manufacturer's instructions including DNase digest. Quality control of total RNA and

224 library integrity was assessed by LabChip Gx Touch 24 (Perkin Elmer). The library was 
227 chemistry and $1 \times 75 b p$ single end setup and the derived values were analyzed for quality,

228 adapter content and duplication rates with FastQC (Available online at:

229 http://www.bioinformatics.babraham.ac.uk/projects/fastqc). Trimming of reads was

230 achieved by employing Trimmomatic version 0.39 after a quality drop below a mean of

231 Q20 in a window of 10 nucleotides (34). Reads of at least 15 nucleotides were approved

232 for subsequent analyses and aligned against the Ensembl human genome version hg38

233 (GRCh38) using STAR 2.6.1d with the parameter "--outFilterMismatchNoverLmax 0.1" to

234 enhance the maximum ratio of mismatches to mapped length to $10 \%(35)$. The number of

235 reads aligning to genes was counted with featureCounts 1.6 .5 from the Subread package

236 (36). Reads overlapping multiple genes or aligning to several genes were excluded, while

237 reads mapping - at least partially - inside exons were accepted and collected per gene.

238 By applying DESeq2 version 1.18.1 differentially expressed genes were identified (37)

239 which were defined by a minimum fold change of \pm 1.5 (log2 +-0.585 ), a maximum

240 Benjamini-Hochberg corrected $p$-value of 0.05 , and a minimum combined mean of 5

241 reads. The Ensemble annotation was enriched with UniProt data (release 24.03.2017)

242 based on Ensembl gene identifiers (Activities at the Universal Protein Resource 243 (UniProt)).

244 RNA expression levels in partial carotid ligation operated age-matched male C57BL/6 245 mice was performed by the laboratory of H.J using 10-week-old and 18-month-old male 246 C57BI/6 mice (The Jackson Laboratory).

\section{Subcellular fractionation}

249 Nuclear (nucleoplasm and chromatin) and cytoplasmic fractions were isolated from 250 untransfected HUVECs. After washing with cold PBS, cells were lysed with cytoplasmic 11 
251 lysis buffer (10 mM Tris ( $\mathrm{pH} 7.5), 150 \mathrm{mM} \mathrm{NaCl}, 0.15 \% \mathrm{NP}-40)$, layered on a sucrose

252 buffer (10 mM Tris ( $\mathrm{pH} 7.5), 150 \mathrm{mM} \mathrm{NaCl}, 24 \%(\mathrm{w} / \mathrm{v})$ sucrose) and centrifuged at $4{ }^{\circ} \mathrm{C}$

253 for $10 \mathrm{~min}$ at $16.000 \mathrm{xg}$. The supernatant (cytoplasmic fraction) was intercepted in TRIzol

254 LS (Thermo Fisher Scientific) for RNA isolation and the pellet was resuspended in glycerol

255 buffer (20 mM Tris ( $\mathrm{pH} 7.9), 75 \mathrm{mM} \mathrm{NaCl}, 0.5 \mathrm{mM}$ EDTA and $0.85 \mathrm{mM}$ DTT, $50 \%$

256 glycerol). Nuclei lysis buffer (10 mM HEPES (pH 7.6), 7.5 mM MgCl2, 0.2 mM EDTA, 0.3

$257 \mathrm{M} \mathrm{NaCl}, 1 \mathrm{M}$ urea, $1 \mathrm{mM}$ DTT, $1 \% \mathrm{NP}-40)$ was added, incubated on ice and centrifuged

258 for $2 \mathrm{~min}$ at $4^{\circ} \mathrm{C}$ and $16.000 \times \mathrm{g}$. The supernatant (nucleoplasm fraction) was resuspended

259 in TRIzol LS. The pellet (chromatin fraction) was resuspended in cold PBS and vigorously

260 vortexed for several seconds to release the RNA. TRIzol LS was added and RNA was

261 isolated using the Direct-zol RNA miniprep kit (Zymo Research). Equal volumes were

262 used for subsequent reverse transcription to ensure comparison of equal cell equivalents.

\section{Growth curve}

265 HUVECs were cultured in 24-well plates (Greiner) and transfected for 24 hours. The cell numbers were counted $0,24,48$ and $72 \mathrm{~h}$ after transfection. For each time point, cells were washed, trypsinized (100 $\mu \mathrm{l}$; Gibco), resuspended in PBS (300 $\mu \mathrm{l})$ and transferred to $1.5 \mathrm{~mL}$ reaction tubes (Eppendorf). The tubes were vortexed and $12 \mu \mathrm{l}$ cell suspension were transferred into Neubauer improved disposable counting chambers (NanoEntek).

270 Cells were counted in 5 large squares ( 4 corner and 1 middle square).The $1.5 \mathrm{~mL}$ reaction

272 determined. Cell number per $\mathrm{mL}$ and total cell number were calculated. 


\section{ECIS and migration assay}

275 Electrical Cell-Substrate Impedance Sensing System (ECIS; Applied BipPhysics) was

276 used to determine the integrity of the endothelial cell barrier, as well as the potential to

277 recover after wounding. As previously described (38), barrier-function was measured by

278 application of an alternating current of $400 \mathrm{~Hz}$. The resulting potential was detected by the

279 ECIS instrument ZO (Applied BioPhysics). The impedance $(\Omega)$ is calculated from the

280 corresponding changes in voltage between electrodes according to Ohm's law (38).

281 Migration analysis was based on the wounding of an intact monolayer by lethal 282 electroporation. The recovery of endothelial cells to form a monolayer was quantified by 283 measuring the impedance at $4000 \mathrm{~Hz}$ (area under the impedance curve).

\section{Caspase $3 / 7$ activity assay}

285 HUVECs were transfected $48 \mathrm{~h}$ before the assay. Cells were transferred to black-walled 96-well plates (Falcon) $4 \mathrm{~h}$ before the assay and incubated with EBM or 200nM Staurosporine (Sigma Aldrich) for $4 \mathrm{~h}$. Caspase 3/7 activity was assayed according to the manufacturer's protocol for ApoOne ${ }^{\circledR}$ Homogenous Caspase 3/7 Assay (Promega). Fluorescence was measured with Glomax Multi plate reader (Promega).

HUVECs were transfected $48 \mathrm{~h}$ before the assay and transferred to gelatin-fibronectincoated specialized 96-well plates (Agilent) $24 \mathrm{~h}$ before the assay. Assay medium was prepared based on Seahorse XF Base Medium (Agilent) and supplemented with L- 
The following protocol was performed according to the manufacturer's instructions

297 including calibration of the Seahorse device, serial injection of Oligomycin, FCCP,

298 Rotenone, Antimycin A and the respective measurement of oxygen consumption rate

299 (OCR) and extracellular acidification rate (ECAR) with Seahorse XFe96 Analyzer

300 (Agilent). For normalization, cells were stained with Hoechst, washed with PBS and

301 luminescence was measured with an ELISA reader. Multiple parameters including basal

302 respiration, ATP-linked respiration and spare respiratory capacity (SRC) were collected in

303 this assay and calculated.

304

305 Static monocyte adhesion assay

306

307

308

309

310

311

312

313

\section{Spheroid assay}

315 again.

HUVECs were transfected $48 \mathrm{~h}$ before the assay and transferred to gelatin-coated blackwalled 96-well plates (Falcon) and stimulated with TNF- $\alpha$ (10 ng/ml; Peprotech) or PBS (Gibco) for $24 \mathrm{~h}$. Directly before start of the assay, THP-1 cells (32) were stained with Hoechst dye (Thermo Fisher) and added to the HUVECs after stringent washing. Baseline fluorescence was measured in an ELISA reader (Promega) following an incubation of 30 min at $37^{\circ} \mathrm{C}, 5 \% \mathrm{CO}_{2}$. Cells were washed with PBS and fluorescence was measured

Endothelial angiogenesis was studied by spheroid sprouting assay in vitro. HUVECs were transfected with siRNAs or LNA GapmeRs for $24 \mathrm{~h}$. Cells were trypsinized and resuspended in a mixture of culture medium and $0.6 \mathrm{gr} / \mathrm{L}$ methylcellulose (Sigma) in a ratio of $80 \%: 20 \%$. Cells were seeded (400 cells per $100 \mu$ l) in a U-bottom-shaped 96 -well 
plate (Greiner) to allow the formation of spheroids for $24 \mathrm{~h}$ at $37^{\circ} \mathrm{C}$. The spheroids were

320 collected, added to methylcellulose (2,4 gr/L) with FBS in a ratio of $80 \%: 20 \%$ (Gibco) and

321 embedded in a collagen type I (Corning) gel containing 3,77 g/L collagen I (Corning, USA),

$32210 \%$ M199 medium (Sigma Aldrich), 0,018 M HEPES (Invitrogen) and $0.2 \mathrm{M} \mathrm{NaOH}$ to

323 adjust $\mathrm{pH}$ to 7,4 . The mixture with the spheroids was allowed to polymerize for 30 minutes

324 in a 24 well plate. Following incubation for $24 \mathrm{~h}$ at $37^{\circ} \mathrm{C}$ with or without VEGFA $(50 \mathrm{ng} / \mathrm{ml}$;

325 Peprotech) the gels were fixed with 10\% formaldehyde (Roth) and microscope images

326 were taken at 10x magnification (AxioVert microscope, Zeiss). The cumulative length of

327 sprouts was quantified using the image analysis software ImageJ.

\section{Statistical analysis}

330 Data are represented by mean \pm standard error of mean (SEM). GraphPad Prism 7 and 3319 were used for statistical analysis. Gaussian distribution was tested using Shapiro-Wilk 332 test. Paired or unpaired Student's t-test or Mann-Whitney tests were performed when 333 comparing two groups. For the comparison of more than two groups Analysis of variance 334 (ANOVA) was applied. A p-value <0.05 was considered significant. 


\section{mouse in endothelial cells and its expression decreases}

\section{strongly during aging}

With the hypothesis that the highest expressed IncRNAs may govern key processes in

ECs, we performed RNA-sequencing to identify novel players in EC biology. Among the

top 10 was TUG1 next to well characterized IncRNAs Metastasis Associated Lung

(NEAT1) and Maternally Expressed 3 (MEG3; Fig 1A). These IncRNAs showed

comparable expression levels as to the EC-specific protein-coding gene Vascular

Endothelial Growth Factor Receptor 2 (VEGFR2; KDR). As previously reported, TUG1 is

ubiquitously expressed in many organs in human and mouse (27). We therefore analyzed

TUG1 expression in various cell types of the cardiovascular system including

cardiomyocytes, fibroblasts, mesenchymal stem cells, smooth muscle cells and pericytes

(Fig 1B). Endothelial cells were further divided into endothelial subtypes derived from

different origins such as dermal, pulmonary and cardiac microvasculature, saphenous

vein and aorta by virtue of their strong heterogeneity. These results showed that TUG1 is

353 ubiquitously expressed across cell types analyzed. TUG1 RNA levels were further

354 analyzed in the context of replicative senescence in human ECs in vitro (Fig 1C), to assess

355 a potential role of TUG1 during aging of the vascular system. TUG1 levels were slightly,

356 but significantly decreased in high passage HUVECs compared to HUVECs in passage 
3, whereas p21 was induced in passage 16 HUVECs as expected. Complementary, Tug1 expression was attenuated in aged (18 months) compared to young mice (10 weeks), to a high extent (Fig 1D). Taken together, TUG1 is highly expressed in endothelial cells and downregulated upon aging in human and mouse. RNA molecules can feature various functions within the cell depending on their subcellular localization (39). Therefore, nuclear (separated into nucleoplasm and chromatin) and cytoplasmic fractions were isolated and analyzed by RT-qPCR (Fig 1E). Differentiation antagonizing non-protein coding RNA (DANCR) and (MALAT1) served as controls. MALAT1 is exclusively associated to

chromatin (40), whereas DANCR is a well characterized transcript known to be mainly cytoplasmic (41), where it is also translated (26). Consistent with previous results obtained within other cell types (42-44), TUG1 was equally distributed across the nucleoplasm, the chromatin, and cytoplasm within ECs, suggesting that TUG1 might incorporate different functions in ECs.

Figure 1: TUG1 is highly expressed in endothelial cells and regulated

\section{by aging in human and mouse.}

373 (A) Top 10 expressed IncRNAs based on transcript counts from HUVEC bulk RNA sequencing data $(n=4)$. TUG1 is highlighted in green. Glyceraldehyde 3-phosphate dehydrogenase (GAPDH) and Kinase Insert Domain Receptor (KDR) were used as controls. (B) RNA expression levels of TUG1 in different human cell types of the cardiovascular system $(n=3)$. Vascular ECs are highlighted by grey bars. AoEC: Aortic 378 ECs, PAEC: Pulmonary Artery ECs, CAEC: Coronary Artery ECs, CMEC: Cardiac 379 Microvascular ECs, DMEC: Dermal Microvascular ECs, PMVEC: Pulmonary 380 Microvascular ECs, SaVEC: Saphenous Vein ECs, HUVEC: Human Umbilical Vein ECs, 17 
DLEC: Dermal Lymphatic ECs, MSC: Mesenchymal Stem Cells, AoAF: Aortic Arterial

Fibroblasts, AoSMC: Aortic Smooth Muscle Cells, CM: Cardiomyocytes (C) TUG1 expression levels in low (P3) vs. high (P16) passage HUVECs as determined by RTqPCR. Expression is relative to GAPDH ( $\mathrm{n}=5-6$; SEM; Mann-Whitney-test). (D) Tug1 expression from bulk RNA-sequencing data of the intima of the carotid arteries of young

Quantification of the expression levels of the IncRNAs Differentiation Antagonizing Non-

Transcript 1 (MALAT1) in subcellular fractions of wild type HUVECs using RT-qPCR

$(n=3)$. Results are expressed as percentages of the subcellular fractions associated to cytoplasm, nucleoplasm and chromatin. Expression is normalized to GAPDH as determined by RT-qPCR.

\section{TUG1 is not involved in proliferation, apoptosis, migration,}

\section{barrier function, mitochondrial function, and inflammation}

\section{under basal conditions in ECs}

To simulate the reduced TUG1 levels in aged human ECs and investigate a potential role of TUG1, HUVECs were transfected with Locked Nucleic Acid (LNA) GapmeRs to reduce the high abundance of the TUG1 transcript in low passage HUVECs. LNA GapmeRs are short single-stranded DNA oligonucleotides that are flanked by LNA nucleotides. Total TUG1 levels were strongly decreased by two different LNA GapmeRs (LNA TUG1_1 $14.46 \% \pm 3.28 \%$; LNA TUG1_2 - 16.40\% $\pm 3.37 \%)$ compared to LNA Ctrl $(100 \% \pm 5.13 \%)$ 
404

405

406

407

408

409

410

411

412

413

414

415

416

417

418

419

420

421

422

423

424

425

426

427

determined. An important hallmark of aging is the reduction of cell proliferation and increased inflammation (14). However, cell turnover - including cell count (Fig 2B) and apoptosis (Fig 2C) - were not changed by loss of TUG1 compared to control. Another ECspecific characteristic addresses barrier function. The method Electric Cell Impedance Sensing (ECIS) analyzes the morphology, which allows to study cell-cell or cell-matrix interactions can be studied. As Fig 2D displays, TUG1 silencing had no effect on either of these interactions. ECIS was also used to determine the migratory capability. For this purpose, a high frequency current was applied and a cell-free area was created by electroporation. Cells from the surrounding area migrate to re-establish a monolayer which can be determined by the change in impedance. The slope of the curve was similar in the control and both TUG1 knockdown conditions, indicating that cells migrated at a similar speed (Fig 2E).

To assess the role of Tug1 in cellular metabolism, parameters assigned to mitochondrial stress (basal respiration, maximal respiration, proton leak, ATP production and spare respiratory capacity) were analyzed after loss of TUG1 using the Seahorse platform. None of the mitochondrial stress characteristics were influenced by loss of TUG1 (Fig 2F). A further characteristic of cardiovascular aging is a low-grade chronic inflammation $(45,46)$. To target this feature, TUG1 was knocked down in HUVECs and the effect on adhesion of monocytes was assessed (Fig 2G). TUG1 manipulation had no effect on monocyte adhesion in untreated and TNF- $\alpha$ stimulated HUVECs. Stimulation with TNF- $\alpha$ was used as a positive control as monocyte adhesion is increased. We tested different chemical stimuli such as oxLDL, $\mathrm{H}_{2} \mathrm{O}_{2}$, VEGFA, TNF- $\alpha$ and Delta-like protein 4 (DII4) and shear stress as mechanical stimulation. TUG1 expression was not changed in response to any of the mentioned stress conditions (S1 Fig). In summary, TUG1 is dispensable for basal 
endothelial function in relation to proliferation, apoptosis, barrier function, migration, mitochondrial function and inflammation.

\section{Figure 2: TUG1 is not important for basal cell turnover, barrier or} mitochondrial function, migration and monocyte adhesion.

(A) - (G) HUVECs were transfected with two LNA GapmeRs against TUG1 - LNA TUG1_1 and LNA TUG1_2 - and LNA Ctrl (10 nM) and (A) expression levels were measured after 48 hours by RT-qPCR. Expression is relative to GAPDH ( $\mathrm{n}=4$; SEM; RM one-way ANOVA with Greenhouse-Geisser correction and Sidak multiple comparison test). (B) Relative cell growth determined from cell count at $0 \mathrm{~h}, 24 \mathrm{~h}, 48 \mathrm{~h}$ and $72 \mathrm{~h}(\mathrm{n}=3$; SEM; RM Two-way ANOVA with Tuckey multiple comparison test). (C) Caspase-3/7 activity was measured by determination of fluorescence with ELISA plate reader $(n=3$; SEM; Oneway ANOVA with Holm-Sidak correction). Staurosporine was taken along as a postive control. (D) Cell-cell interactions $(\mathrm{Rb})$ and cell-matrix-interactions $(\alpha)$ were measured by Electric Cell Impedance Sensing (ECIS; $\mathrm{n}=3$; SEM; Kruskal-Wallis-test with Dunn's correction). (E) Determination of re-establishment of monolayer after wounding using ECIS ( $n=3$; SEM; One-way ANOVA with Holm-Sidak multiple comparison test). (F) Seahorse mitochondrial stress test assessing multiple mitochondrial characteristics via measurement of changes in Oxygen Consumption Rate (OCR) after serial injection of Oligomycin, Carbonyl cyanide-4 (trifluoromethoxy) phenylhydrazone (FCCP) and Rotenone A/Antimycin ( $\mathrm{n}=3$; SEM; One-way ANOVA with Holm-Sidak multiple comparison test. One representative experiment displaying the changes of OCR throughout the progress of the Seahorse mitochondrial stress test assay. (G) Assessment 
451 of monocyte adhesion with and without TNF- $\alpha$ stimulation. ( $n=3$; SEM; Two-way ANOVA 452 with Tuckey multiple comparison test).

\section{TUG1 IncRNA is not required for basal sprouting, but relevant}

\section{5 for VEGFA-stimulated sprouting in vitro}

Advanced aging is often accompanied by a decline in angiogenesis resulting in increased

cardiovascular morbidity and mortality $(47,48)$. Therefore, endothelial cell sprouting was assessed in an in vitro angiogenesis assay. The loss of TUG1 had no effect on human EC sprouting under basal conditions in vitro (Fig 3A). Conversely, TUG1 knockdown slightly, but significantly, reduced cumulative sprout length after VEGFA-stimulation. Moreover, RNA sequencing following GapmeR-mediated silencing of HUVECs (GapmeR Control vs. GapmeR TUG1) resulted in only minor changes in gene expression, despite a robust reduction in TUG1 levels (Fig 3B). Some of the significantly regulated targets from the RNA-seq dataset were further analyzed by RT-qPCR (S2 Fig). None of these were robustly regulated by both GapmeRs. Hence, TUG1 does not affect the transcriptional profile in ECs. This supported the results from the EC-specific functional assays, because TUG1 silencing had no impact on the described characteristics.

Additionally, siRNAs were used to attenuate TUG1 transcript levels in HUVECs. In more detail, two siRNAs resulted in a knockdown efficiency of more than 50\% (siTUG1_1 37.62\% \pm 9.14\%; siTUG1_2-45.25\% $\pm 5.78 \%$; Fig 3C). Basal sprouting was not changed

471 after siRNA-mediated TUG1 knockdown, whereas VEGFA-stimulated sprout length was 472 reduced by TUG1 reduction (Fig 3D). In conclusion, TUG1 is not relevant for the regulation 473 of basal sprouting, but TUG1 knockdown in combination with VEGFA stimulation 
474 decreased cumulative sprout length to a small extent as compared to VEGFA stimulation

475 alone.

\section{Figure 3: TUG1 influences VEGFA-stimulated sprouting.}

478 (A) Quantification of cumulative sprout length by in vitro spheroid-assay after LNA 479 GapmeR-mediated TUG1 knockdown under basal conditions or with VEGFA stimulation 480 (50 ng/ml for $24 \mathrm{~h}$ ) in HUVECs. Representative images show the extent of sprouting as compared to $200 \mu \mathrm{m}$ size bar ( $\mathrm{n}=5$; SEM; RM two-way ANOVA with Geisser-Greenhouse correction and Holm-Sidak multiple comparison test).

(B) Volcano plot of deregulated genes (log2 fold change vs. -log10 p-value) based on HUVEC bulk RNA-sequencing data (LNA Ctrl vs. LNA TUG1; $\mathrm{n}=3$ vs. 3). TUG1 is represented by green dot. (C) TUG1 expression levels in HUVECs 48h after siRNA transfection using RT-qPCR. Expression

487 Quantification of cumulative sprout length by in vitro spheroid-assay after siRNA-mediated TUG1 knockdown under basal conditions or with VEGFA stimulation (50 ng/ml for $24 \mathrm{~h}$ ) in HUVECs. Representative images show the extent of sprouting as compared to $200 \mu \mathrm{m}$ size bar ( $\mathrm{n}=4$; SEM; RM two-way ANOVA with Geisser-Greenhouse correction and Holm-Sidak multiple comparison test).

\section{TUG1 protein can be overexpressed in ECs, but is not involved}

\section{in regulation of angiogenic sprouting}

495 TUG1 was recently described to encode a protein with a length of 153 amino acids (27,

496 26). To see whether this predicted protein can be expressed in, and perhaps has a 
497

498

499

500

501

502

503

504

505

506

507

508

509

510

511

512

513

514

function in ECs, different constructs were generated for lentiviral overexpression (OE) in HUVECs. Three different inserts were subcloned from pEF1a plasmids (26) into the pLenti4-V5 backbone (Fig 4A).

The construct named pLenti4-V5_hsTUG1_IncRNAshort contains almost the entire human TUG1 IncRNA transcript, including parts of the endogenous 5'-UTR, the TUG1 protein open reading frame (ORF) with its non-canonical start codon (CTG) and a shortened 3'-UTR. The pLenti4-V5_hsTUG1_CTGmut plasmid differs from the former only by the replacement of the non-canonical start codon of the TUG1 ORF by TAG, which should prevent protein translation but leaves the remaining IncRNA intact. In contrast to the previous two, pLent4-V5_hsTUG1_CDS only contains the information for the TUG1 protein in form of the codon-optimized TUG1 ORF, but not for the IncRNA. In all plasmids a 3xFLAG-tag is inserted at the C-terminus of the TUG1 ORF resulting in a TUG13xFLAG-tag fusion protein in the case of translation.

Using these different constructs enables assigning certain effects to the IncRNA, the protein or both. After lentiviral transduction, RNA levels were expectedly strongly increased for all three constructs as determined by RT-qPCR (Fig 4B). Protein levels were assessed using anti-FLAG antibodies that target the C-terminal tag attached to the different proteins described in Fig 4A. As expected, the TUG1-3xFLAG-tag fusion protein was translated from the hsTUG1_IncRNAshort construct in HUVECs, whereas the CTG mutation in hsTUG1_CTGmut abolished TUG1 protein production (Fig 4C). The protein encoded by the hsTUG1_CDS construct was expressed to a smaller extent. Functionally, none of the constructs resulted in significant changes of sprout length in angiogenic sprouting assays in vitro compared to the control pLenti4-V5_mock (Fig 4D), neither under basal conditions nor after stimulation with VEGFA. 
521 In summary, although the TUG1 protein could be translated following exogenous

522 overexpression in HUVECs, it appeared not to be relevant for controlling angiogenesis.

523 These results indicate that, regardless of TUG1's translation potential, TUG1 is unlikely to 524 regulate endothelial cell function in vitro.

526 Figure 4. TUG1 protein can be overexpressed in HUVECs, but is not 527 involved in sprouting.

528 (A) Scheme of three pLenti4-V5 plasmids with different inserts for the TUG1 IncRNA and 529 protein coding open reading frame (ORF). PLenti4-V5_hsTUG1_IncRNAshort with the 530 non-canconical start codon CTG representing the wild type sequence containing the 531 information for the protein and the IncRNA, pLenti4-V5_hsTUG1_CTGmut with mutated 532 start codon to stop codon (TAG) containing the information for the IncRNA only and 533 pLenti4-V5_hsTUG1_CDS containing the codon optimized ORF for the TUG1 protein 534 only. 5'- and 3'-untranslated region (UTR) indicated in green, ORF indicated in blue and 535 C-terminal 3xFLAG-tag indicated in orange. Start of ORF sequence underlined and 536 highlighted in blue. (B) RNA levels following lentiviral overexpression of the three different 537 TUG1 proteins in HUVECs as determined by RT-qPCR ( $n=4$; SEM; ratio paired t-test). (C) 538 Acquisition of TUG1 protein translation after exogenous lentiviral overexpression of TUG1 539 proteins by Western Blot using anti-FLAG antibody ( $n=4$; SEM; RM one-way ANOVA with 540 Geisser-Greenhouse correction and Holm-Sidak multiple comparison test). (D) 541 Quantification of cumulative sprout length by in vitro spheroid-assay after lentiviral 542 overexpression of TUG1 protein constructs under basal conditions or with VEGFA 543 stimulation (50 ng/ml for $24 \mathrm{~h}$ ) in HUVECs. Representative images show the extent of 
bioRxiv preprint doi: https://doi.org/10.1101/2022.02.27 482212; this version posted February 28, 2022. The copyright holder for this preprint

(which was not certified by peer review) is the author/funder, who has granted bioRxiv a license to display the preprint in perpetuity. It is made available under aCC-BY 4.0 International license.

544 sprouting as compared to $200 \mu \mathrm{m}$ size bar ( $\mathrm{n}=4$; SEM; RM two-way ANOVA with Geisser-

545 Greenhouse correction and Holm-Sidak multiple comparison test).

546 


\section{Discussion}

548 This study identified that TUG1 expression was attenuated by aging in human and mouse

549 ECs. TUG1 silencing had no effect on basal EC function including proliferation, apoptosis, 550 barrier function, migration, mitochondrial function and monocyte adhesion, while VEGFA551 stimulated sprouting was decreased significantly. Furthermore, TUG1 did not influence 552 the transcriptional profile. The TUG1 proteins (encoded by the hsTUG1_IncRNAshort and 553 hsTUG1_CDS constructs) were translated in HUVECs following lentiviral overexpression, 554 while overexpression of the construct with a mutated start codon (hsTUG1_CTGmut) did 555 not result in detectable TUG1 protein. The TUG1 proteins did not regulate basal or VEGFA 556 stimulated angiogenic sprouting in vitro.

TUG1 is an interesting IncRNA because of a remarkable combination of features: TUG1 was highly and ubiquitously expressed in multiple cell types and conserved among many different species (27). Our results further showed an equal distribution in nucleus and cytoplasm (Fig 1E) and a regulation by aging in human (Fig 1C) and mouse (Fig 1D). intima, endothelial dysfunction and increased vascular inflammation (14). This functional decline of ECs is caused by oxidative stress, epigenetic changes, endothelial dysfunction and genomic instability (45). We expected alterations in at least some of these 566 characteristics following silencing of TUG1. Therefore, GapmeRs were used to target all TUG1 transcripts (nuclear or cytoplasmic; Fig 2A) for the simulation of aged ECs.

568 Unexpectedly, loss of TUG1 did not change any phenotypic parameters related to aging 569 in ECs under basal conditions (Fig 2B, 2C, 2D, 2E, 2F and 2G). Instead, TUG1 
570 manipulation only resulted in a slight decrease of VEGFA-stimulated sprouting by using

571 GapmeRs or siRNAs (Fig 3A and D). Manipulation of previously studied IncRNAs in loss-

572 of-function studies resulted in stronger attenuation of angiogenic sprouting also at basal

573 level $(49,50,1)$. Thus, the absence of effects on basal EC function after loss of TUG1

574 represents a novelty. The dispensability of TUG1 under basal conditions was further

575 underlined by the results from bulk RNA-sequencing of control vs. TUG1 knockdown.

576 TUG1 was the most robustly downregulated gene, while only very few differentially

577 regulated genes resulted from the analysis of bulk RNA-sequencing data (S2 Fig).

578 Consequently, TUG1 did not contribute to transcriptional regulation in HUVECs under

579 basal conditions. In vivo data showed that global Tug1 knockout had no phenotype except

580 for male infertility caused by impaired spermatogenesis with defects in number of sperms

581 and abnormal sperm morphology (27). Consequently, we do not expect a phenotype in

582 angiogenesis.

584 Interestingly, the regulation by aging was the only significant upstream effect to be 585 involved in the regulation of TUG1 expression. Neither oxidized low density lipoprotein 586 (oxLDL) nor hydrogen peroxide $\left(\mathrm{H}_{2} \mathrm{O}_{2}\right)$ - both oxidative stressors - influenced TUG1 RNA 587 levels (S1A and S1B Figs). Furthermore, activating stimuli such as VEGFA and TNF- $\alpha$ or 588 mechanic forces represented by shear stress did not change TUG1 expression in 589 HUVECs (S1C, S1D and S1E Figs). TUG1 was previously described to be induced by 590 Notch1 which is accompanied by promotion of self-renewal of glioma stem cells (21). Delta 591 Like Canonical Notch Ligand 4 (D/l4) - which is an established activator of the Notch 592 pathway in endothelial cells - was not able to induce TUG1 expression in HUVECs (S1F 593 Fig). 
595 Even though we did not find evidence for a role of TUG1 in ECs under normal culture conditions, TUG1 might play a role under certain stress stimuli. The high levels of TUG1

597 transcript might serve as a backup for certain stress responses. This was further supported by the findings of Dumbovic et al. (44): Intron retention in the TUG1 transcript drives nuclear compartmentalization and the authors hypothesize that this might indeed serve as a system for buffering the TUG1 transcript in particular stress conditions. In addition, TUG1 is involved in diabetic retinopathy in mice (6) and many types of cancer via a nuclear or cytoplasmic function $(21,51,30,52,53)$. Taken together, these findings hint towards a cell- or context-specific function of TUG1.

Recently, translation of a TUG1 protein was revealed by which the TUG1 gene might exert an additional function (26). Three different lentiviral constructs were generated depicting 607 the TUG1 transcript (hsTUG1_CTGmut), the TUG1 protein (hsTUG1_CDS) or both 608 (hsTUG1_IncRNAshort) with a C-terminal FLAG-tag. None of the overexpressed 609 constructs changed angiogenic sprouting in vitro under basal or VEGFA-stimulated conditions significantly. According to Lewandowski et al. (27), the trans-based function of

611 the TUG1 IncRNA is negligible, whereas the TUG1 protein is involved in the regulation of 612 mitochondrial bioenergetics. We could not identify a role of TUG1 in mitochondrial function 613 in ECs (Figure 2F).

615 In summary, we show that despite a high abundance and conservation of the IncRNA 616 TUG1 and the encoded protein, both are not essential for basal EC function. The small, 
bioRxiv preprint doi: https://doi.org/10.1101/2022.02.27.482212; this version posted February 28, 2022. The copyright holder for this preprint

(which was not certified by peer review) is the author/funder, who has granted bioRxiv a license to display the preprint in perpetuity. It is made available under aCC-BY 4.0 International license.

617 but significant, contribution of the TUG1 IncRNA to VEGF-induced endothelial cell

618 sprouting, likely only influences endothelial cell function to a minor extent, if any. 


\section{Literature Cited}

620 1. Hofmann P, Sommer J, Theodorou K, Kirchhof L, Fischer A, Li Y et al. Long non-coding RNA H19

621 regulates endothelial cell aging via inhibition of STAT3 signalling. Cardiovasc Res 2019; 115(1):230-42.

622 2. Boon RA, Hofmann P, Michalik KM, Lozano-Vidal N, Berghäuser D, Fischer A et al. Long Noncoding RNA

623 Meg3 Controls Endothelial Cell Aging and Function: Implications for Regenerative Angiogenesis. J Am Coll

624 Cardiol 2016; 68(23):2589-91.

625 3. Guo X, Chang Q, Pei H, Sun X, Qian X, Tian C et al. Long Non-coding RNA-mRNA Correlation Analysis

626 Reveals the Potential Role of HOTAIR in Pathogenesis of Sporadic Thoracic Aortic Aneurysm. Eur J Vasc

627 Endovasc Surg 2017; 54(3):303-14.

628 4. Yari M, Bitarafan S, Broumand MA, Fazeli Z, Rahimi M, Ghaderian SMH et al. Association between Long

629 Noncoding RNA ANRIL Expression Variants and Susceptibility to Coronary Artery Disease. Int J Mol Cell

630 Med 2018; 7(1):1-7.

631 5. Balas MM, Johnson AM. Exploring the mechanisms behind long noncoding RNAs and cancer.

632 Noncoding RNA Res 2018; 3(3):108-17.

633 6. Long J, Badal SS, Ye Z, Wang Y, Ayanga BA, Galvan DL et al. Long noncoding RNA Tug1 regulates

634 mitochondrial bioenergetics in diabetic nephropathy. J Clin Invest 2016; 126(11):4205-18.

635 7. Tan CSH, Gay EMQ, Ngo WK. Is age a risk factor for diabetic retinopathy? Br J Ophthalmol 2010;

$63694(9): 1268$.

637 8. Wang Y, Wang X, Wang Y-X, Ma Y, Di Y. The Long-Noncoding RNA TUG1 Regulates Oxygen-Induced

638 Retinal Neovascularization in Mice via MiR-299. Invest Ophthalmol Vis Sci 2022; 63(1):37. 
639 9. Cai H, Liu X, Zheng J, Xue Y, Ma J, Li Z et al. Long non-coding RNA taurine upregulated 1 enhances

640 tumor-induced angiogenesis through inhibiting microRNA-299 in human glioblastoma. Oncogene 2017;

$64136(3): 318-31$.

642 10. Yu X, Hu L, Li S, Shen J, Wang D, Xu R et al. Long non-coding RNA Taurine upregulated gene 1

643 promotes osteosarcoma cell metastasis by mediating HIF-1 $\alpha$ via miR-143-5p. Cell Death Dis 2019;

644 10(4):280.

645 11. Mc Namara K, Alzubaidi H, Jackson JK. Cardiovascular disease as a leading cause of death: How are

646 pharmacists getting involved? Integr Pharm Res Pract 2019; 8:1-11.

647 12. Timmis A, Townsend N, Gale C, Grobbee R, Maniadakis N, Flather M et al. European Society of

648 Cardiology: Cardiovascular Disease Statistics 2017. Eur Heart J 2018; 39(7):508-79.

649 13. Heidenreich PA, Trogdon JG, Khavjou OA, Butler J, Dracup K, Ezekowitz MD et al. Forecasting the

650 future of cardiovascular disease in the United States: A policy statement from the American Heart

651 Association. Circulation 2011; 123(8):933-44.

652 14. Lakatta EG, Levy D. Arterial and cardiac aging: Major shareholders in cardiovascular disease

653 enterprises: Part I: aging arteries: a "set up" for vascular disease. Circulation 2003; 107(1):139-46.

654 15. Moriya J, Minamino T. Angiogenesis, Cancer, and Vascular Aging. Front Cardiovasc Med 2017; 4:65.

655 16. Chung AS, Ferrara N. Developmental and pathological angiogenesis. Annu Rev Cell Dev Biol 2011;

$656 \quad 27: 563-84$.

657 17. Klagsbrun M, D'Amore PA, editors. Angiogenesis: Biology and pathology. Cold Spring Harbor, N.Y:

658 Cold Spring Harbor Laboratory Press; 2011.

659 18. Puro DG, Kohmoto R, Fujita Y, Gardner TW, Padovani-Claudio DA. Bioelectric impact of pathological

660 angiogenesis on vascular function. Proc Natl Acad Sci U S A 2016; 113(35):9934-9. 
20. Lee JT, Bartolomei MS. X-inactivation, imprinting, and long noncoding RNAs in health and disease.

663 Cell 2013; 152(6):1308-23.

21. Katsushima K, Natsume A, Ohka F, Shinjo K, Hatanaka A, Ichimura N et al. Targeting the Notchregulated non-coding RNA TUG1 for glioma treatment. Nat Commun 2016; 7:13616.

22. Rinn JL, Kertesz M, Wang JK, Squazzo SL, Xu X, Brugmann SA et al. Functional demarcation of active and silent chromatin domains in human HOX loci by noncoding RNAs. Cell 2007; 129(7):1311-23. dihydrofolate reductase gene by a non-coding interfering transcript. Nature 2007; 445(7128):666-70. MALAT1 regulates alternative splicing by modulating SR splicing factor phosphorylation. Mol Cell 2010;

$672 \quad 39(6): 925-38$.

673 25. Tsuiji H, Yoshimoto R, Hasegawa Y, Furuno M, Yoshida M, Nakagawa S. Competition between a 674 noncoding exon and introns: Gomafu contains tandem UACUAAC repeats and associates with splicing

675 factor-1. Genes Cells 2011; 16(5):479-90.

676 26. van Heesch S, Witte F, Schneider-Lunitz V, Schulz JF, Adami E, Faber AB et al. The Translational

677 Landscape of the Human Heart. Cell 2019; 178(1):242-260.e29.

679 locus is essential for male fertility. Genome Biol 2020; 21(1):237. 

tumor growth and angiogenesis in hepatoblastoma. Cell Death Dis 2016; 7(6):e2278.

684 30. Khalil AM, Guttman M, Huarte M, Garber M, Raj A, Rivea Morales D et al. Many human large 685 intergenic noncoding RNAs associate with chromatin-modifying complexes and affect gene expression.

686 Proc Natl Acad Sci U S A 2009; 106(28):11667-72.

687 31. Makarewich CA, Olson EN. Mining for Micropeptides. Trends Cell Biol 2017; 27(9):685-96.

32. Drexler HG, Dirks W, MacLeod RA, Quentmeier H, Steube, KG, Uphoff, CC. DSMZ Catalogue of Human and Animal Cell Lines. Available from: URL: ww.dsmz.de. efficient gene delivery in vivo. Nat Biotechnol 1997; 15(9):871-5. Bioinformatics 2014; 30(15):2114-20. aligner. Bioinformatics 2013; 29(1):15-21. reads to genomic features. Bioinformatics 2014; 30(7):923-30. quantification of endothelial proliferation, barrier function, and motility. J Vis Exp 2014; (85). 220(2). 
MALAT1 regulates endothelial cell function and vessel growth. Circ Res 2014; 114(9):1389-97.

41. Mukherjee N, Calviello L, Hirsekorn A, Pretis S de, Pelizzola M, Ohler U. Integrative classification of

707 human coding and noncoding genes through RNA metabolism profiles. Nat Struct Mol Biol 2017;

$708 \quad 24(1): 86-96$.

42. van Heesch S, van Iterson M, Jacobi J, Boymans S, Essers PB, Bruijn E de et al. Extensive localization of long noncoding RNAs to the cytosol and mono- and polyribosomal complexes. Genome Biol 2014; 15(1):R6.

43. Cabili MN, Dunagin MC, McClanahan PD, Biaesch A, Padovan-Merhar O, Regev A et al. Localization

713 and abundance analysis of human IncRNAs at single-cell and single-molecule resolution. Genome Biol

$714 \quad 2015 ; 16: 20$.

715

44. Dumbović G, Braunschweig U, Langner HK, Smallegan M, Biayna J, Hass EP et al. Nuclear compartmentalization of TERT mRNA and TUG1 IncRNA is driven by intron retention. Nat Commun 2021; 12(1):3308

45. Paneni F, Diaz Cañestro C, Libby P, Lüscher TF, Camici GG. The Aging Cardiovascular System: Understanding It at the Cellular and Clinical Levels. J Am Coll Cardiol 2017; 69(15):1952-67.

46. López-Otín C, Blasco MA, Partridge L, Serrano M, Kroemer G. The hallmarks of aging. Cell 2013; 153(6):1194-217.

47. Ungvari Z, Tarantini S, Kiss T, Wren JD, Giles CB, Griffin CT et al. Endothelial dysfunction and angiogenesis impairment in the ageing vasculature. Nat Rev Cardiol 2018; 15(9):555-65.

724 48. Lähteenvuo J, Rosenzweig A. Effects of aging on angiogenesis. Circ Res 2012; 110(9):1252-64. 
727 50. Pham TP, Bink DI, Stanicek L, van Bergen A, van Leeuwen E, Tran Y et al. Long Non-coding RNA Aerrie

728 Controls DNA Damage Repair via YBX1 to Maintain Endothelial Cell Function. Front Cell Dev Biol 2020;

$7298: 619079$.

730 51. Huang M-D, Chen W-M, Qi F-Z, Sun M, Xu T-P, Ma P et al. Long non-coding RNA TUG1 is up-regulated

731 in hepatocellular carcinoma and promotes cell growth and apoptosis by epigenetically silencing of KLF2.

732 Mol Cancer 2015; 14:165.

733 52. Niu Y, Ma F, Huang W, Fang S, Li M, Wei T et al. Long non-coding RNA TUG1 is involved in cell growth

734 and chemoresistance of small cell lung cancer by regulating LIMK2b via EZH2. Mol Cancer 2017; 16(1):5.

735 53. Tan J, Qiu K, Li M, Liang Y. Double-negative feedback loop between long non-coding RNA TUG1 and

736 miR-145 promotes epithelial to mesenchymal transition and radioresistance in human bladder cancer

737 cells. FEBS Lett 2015; 589(20 Pt B):3175-81. 


\section{Author contributions}

740 ATG designed and performed the experiments, analyzed the data, and drafted the manuscript.

741 SK, KT, JFS and LS provided technical, conceptual advice, and performed experiments. SG

742 performed and analyzed RNA-sequencing data. TA analyzed RNA-sequencing data. SK and HJ

743 performed research. SD provided technical, conceptual advice. SvH and NH provided technical,

744 conceptual advice, and provided samples. RAB supervised the project, designed experiments,

745 analyzed data, handled funding, and drafted the manuscript. All authors contributed to the article

746 and approved the submitted version. 


\section{Figure Legends for Supplemental Figures}

\section{S1 Fig: TUG1 is not regulated by EC activation, induction of}

\section{quiescence, oxidative stress or inflammation.}

751 TUG1 RNA levels were measured by RT-qPCR after stimulation with (A) $50 \mu \mathrm{g} / \mathrm{ml}$ oxLDL for ( $n=3$; SEM; unpaired t-test; Hes Family BHLH Transcription Factor 1 (HES1) served as a Ctrl). Expression is normalized to GAPDH or TBP as determined by RT-qPCR.

\section{S2 Fig: RT-qPCR-based confirmation of RNA-sequencing results}

\section{using both LNA GapmeRs against TUG1.}

HUVECs were transfected with two LNA GapmeRs against TUG1 - LNA TUG1_1 and LNA

TUG1_2 - and LNA Ctrl and expression levels of (A) VAMP4, (B) TOR1AIP2, (C) KAT6B and

(D) ABCA1 were measured after 48 hours by RT-qPCR. Expression is relative to P0 ( $n=4$; 
A

\section{Scheme of pLenti4-V5 constructs}

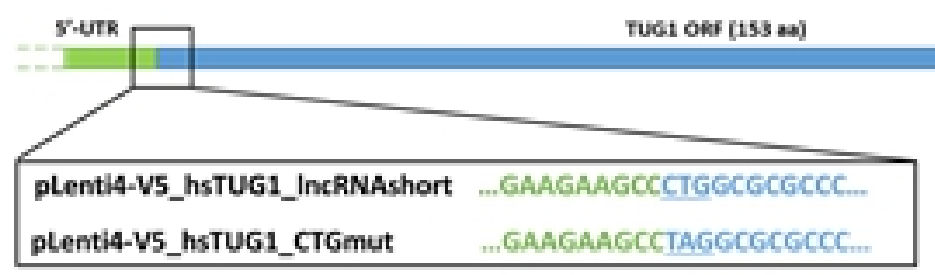

BafLG Y-UTR

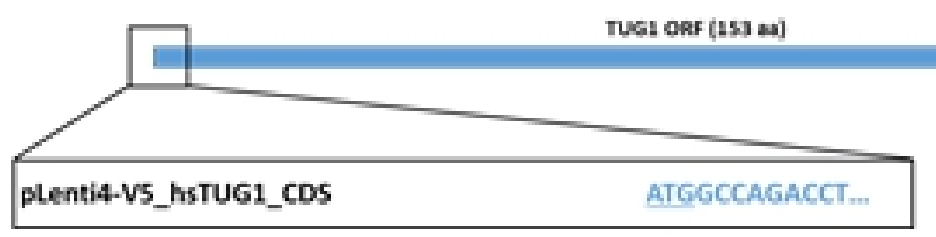

B

RNA levels of pLenti4-V5 constructs

bioRxiv preprint doi: https://doi org/10.1101/2022.02:27.48 (which was not certified by peer review) is the author/funder, who has granted bioRxiv a license to display the preprint in perpetuity. It is made available under ACC-BY 4.0 International license.
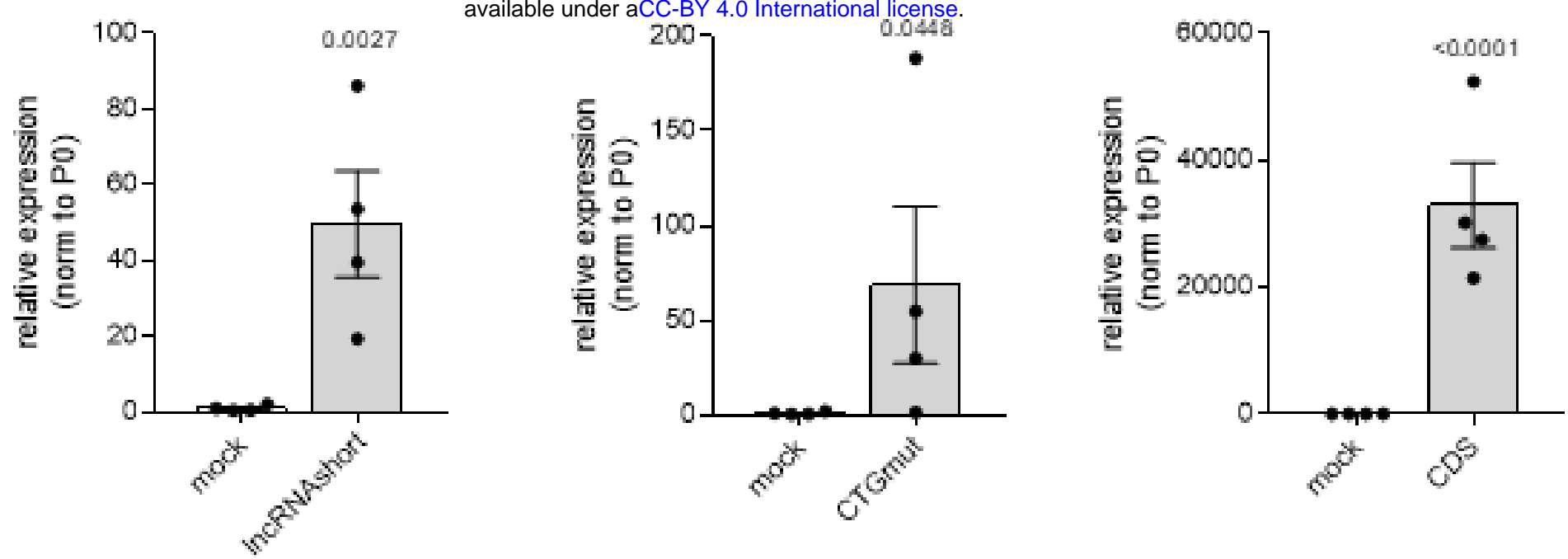

C

\section{TUG1 peptide expression}

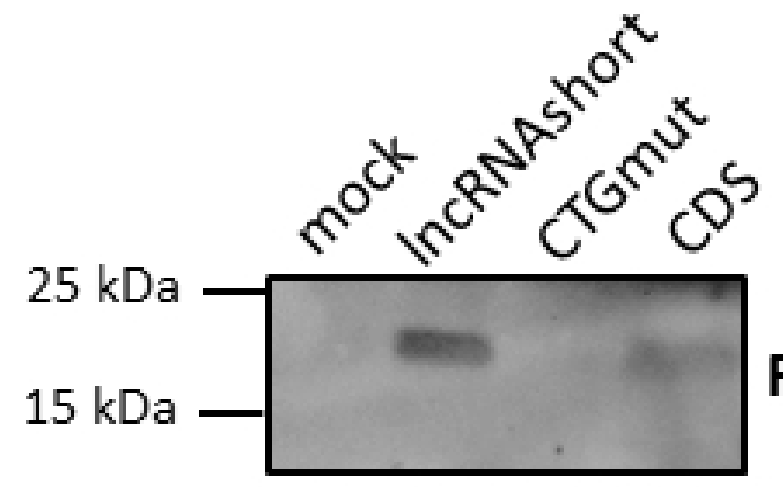

FLAG
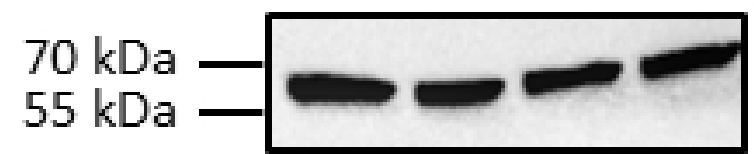

$\beta$-Tubulin

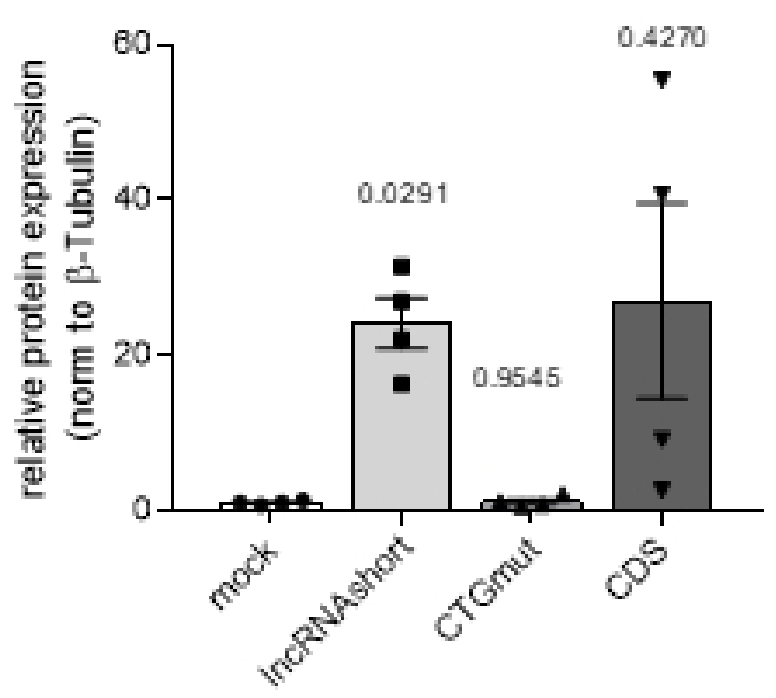

D

\section{Sprouting angiogenesis (TUG1 overexpression)}
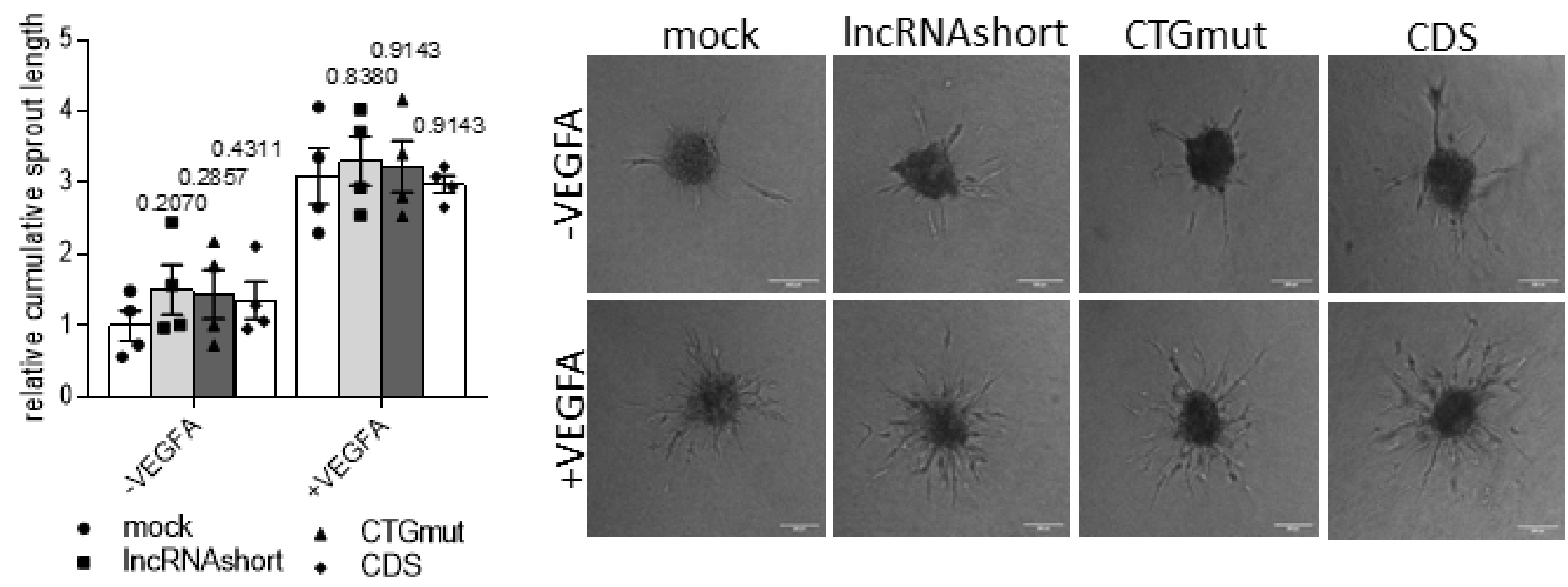
A

Top 10 expressed IncRNAs in HUVECs

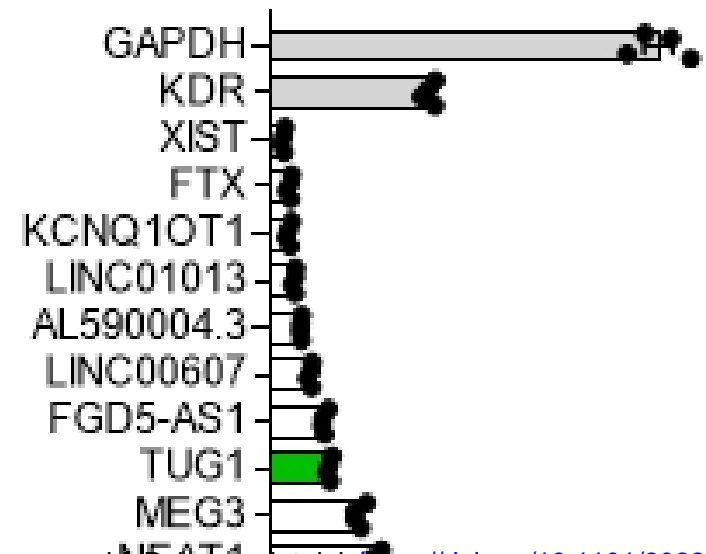

MEG3

bidExA Treptint doi:

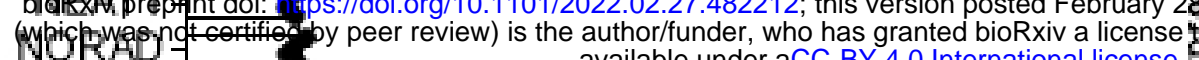

MALAT1

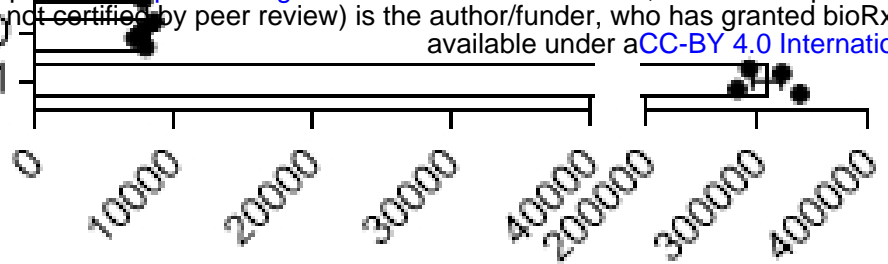

counts

C

\section{TUG1 and p21 expression in} replicative senescence
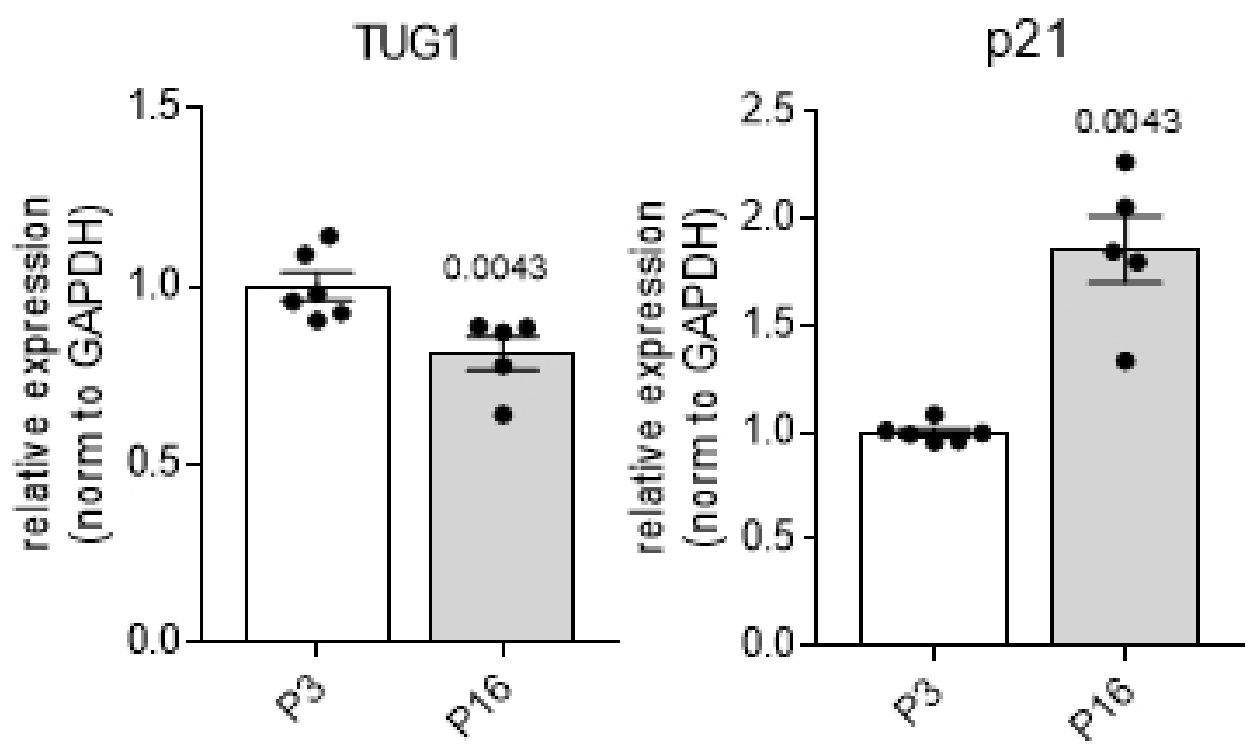

TUG1 expression in different cells of the cardiovascular system

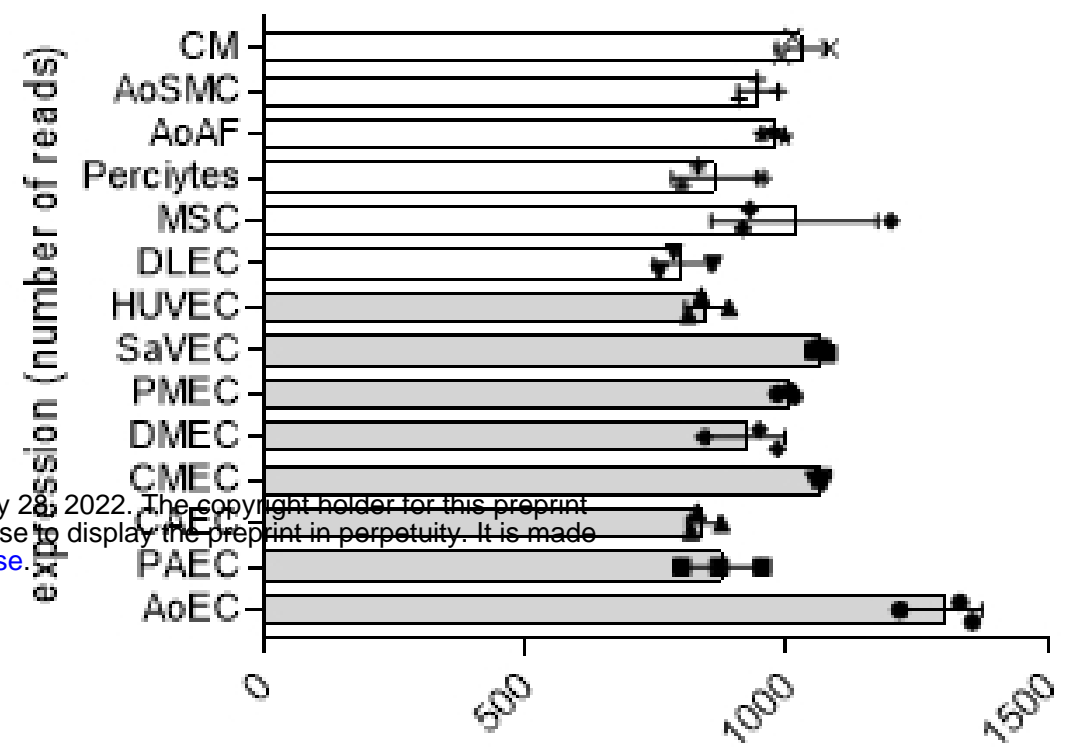

D

Tug1 expression in mouse endothelial cells

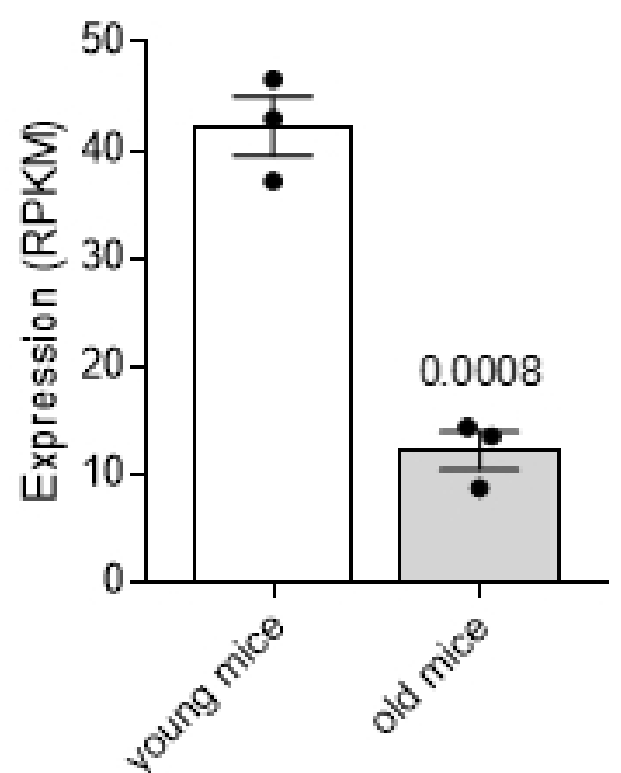

E

Subcellular fractionation

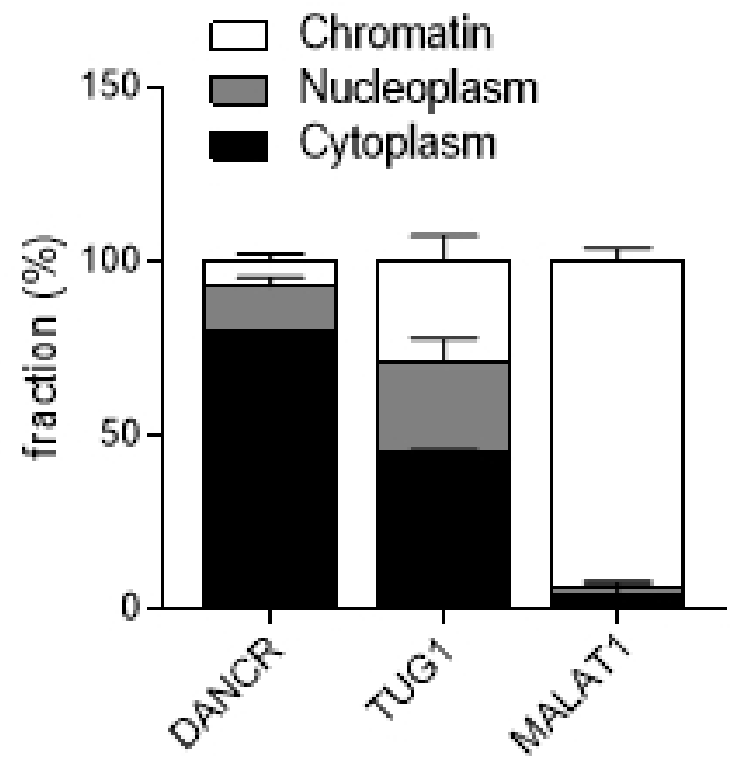




\section{Figure 2}

A

TUG1 expression

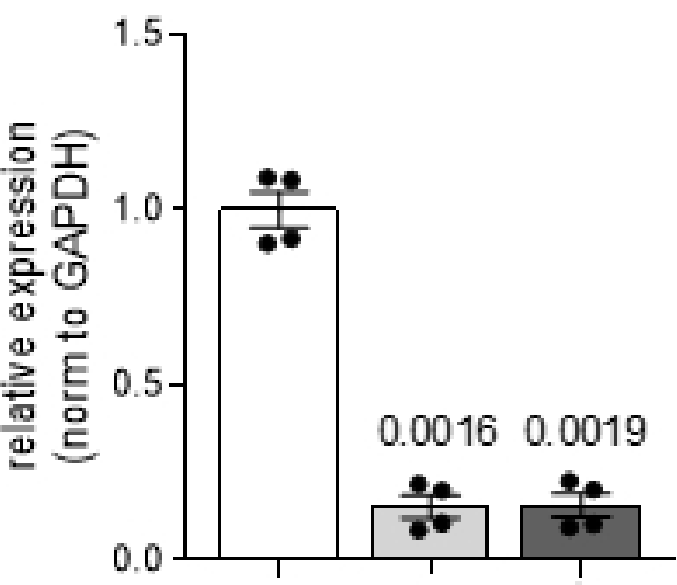

B

Growth curve
C

Caspase $3 / 7$ activity

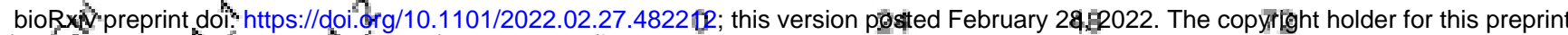
(whiefl was not-certified by perer'review) is the author/funder, who has granted bioRxiv a license to display the preprint in perpetuity. It is made available under aCC-BY 4.0 Internationdilingens(h) whiea was notgertified by eger $v^{3}$

D

$$
\text { Cell-cell }
$$

interactions

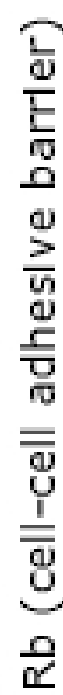

Cell-matrix interactions

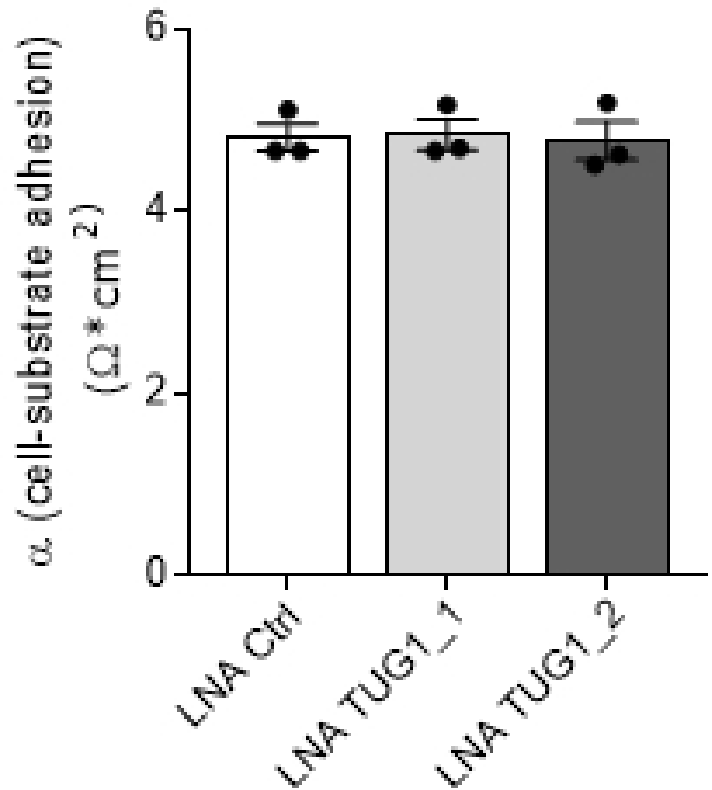

$\mathbf{F}$

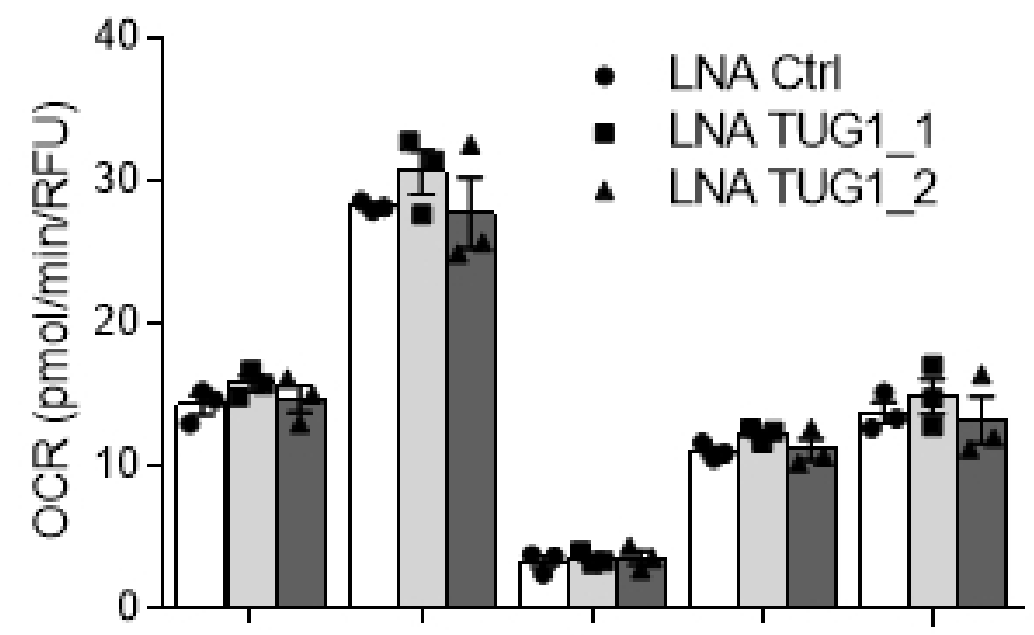

E

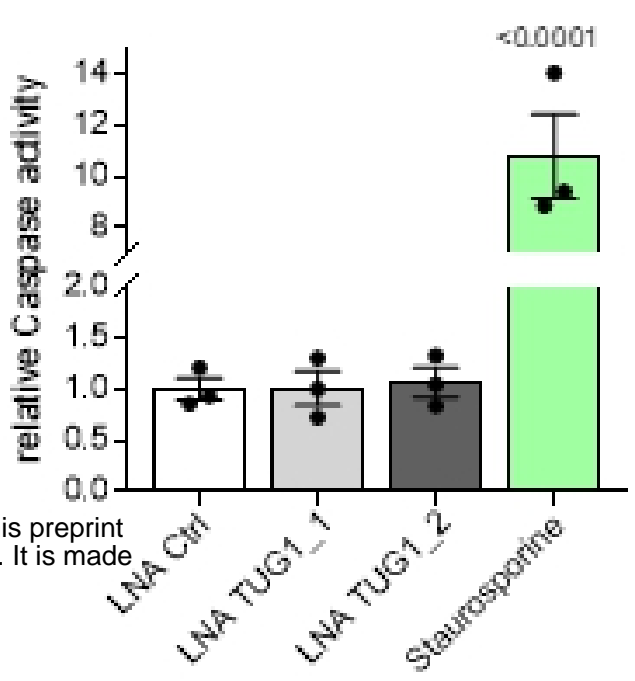

Cell migration

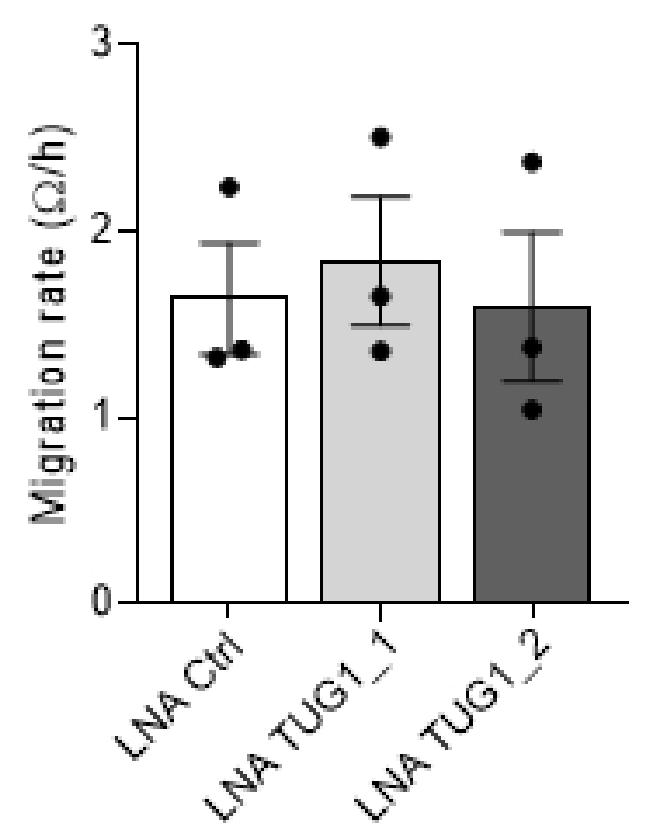

G

\section{Static}

monocyte adhesion

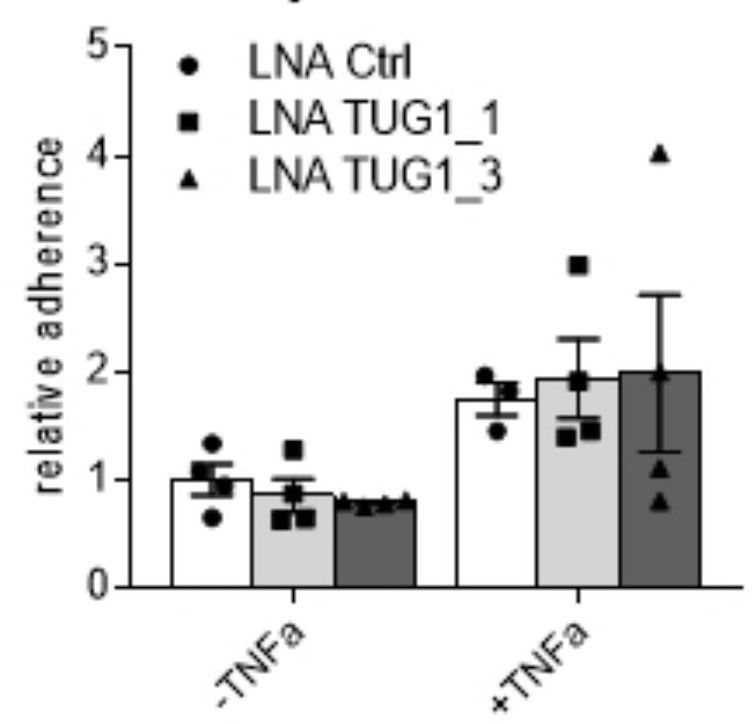

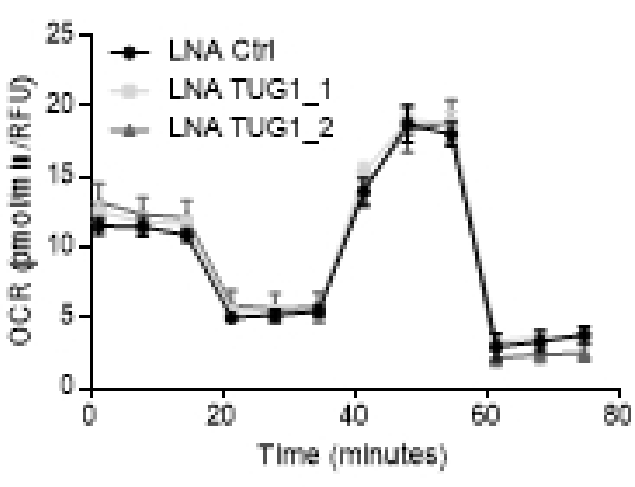


A

Sprouting angiogenesis (LNA GapmeR)

C

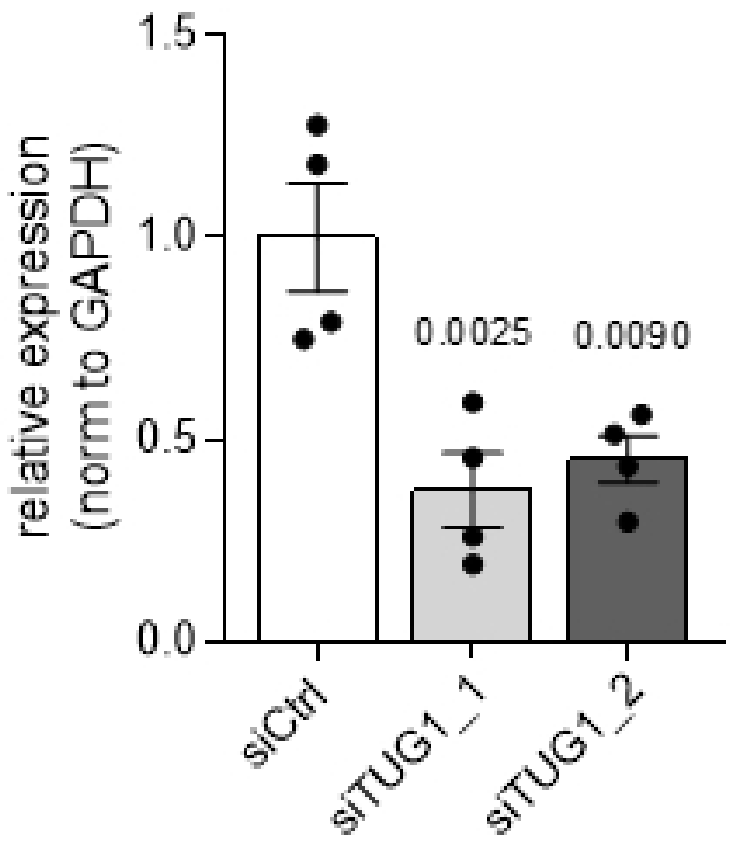

TUG1 expression

D

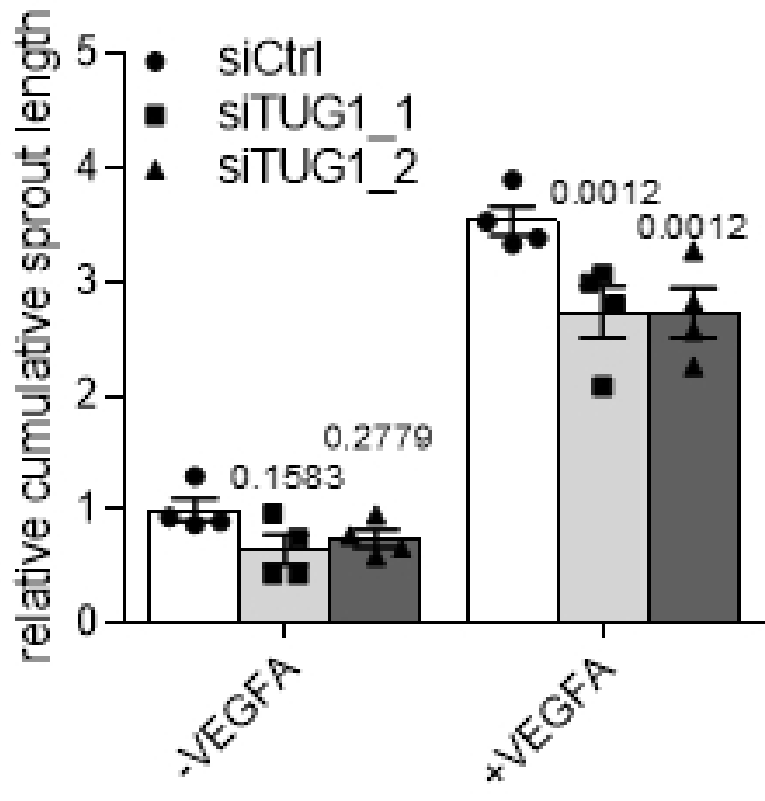

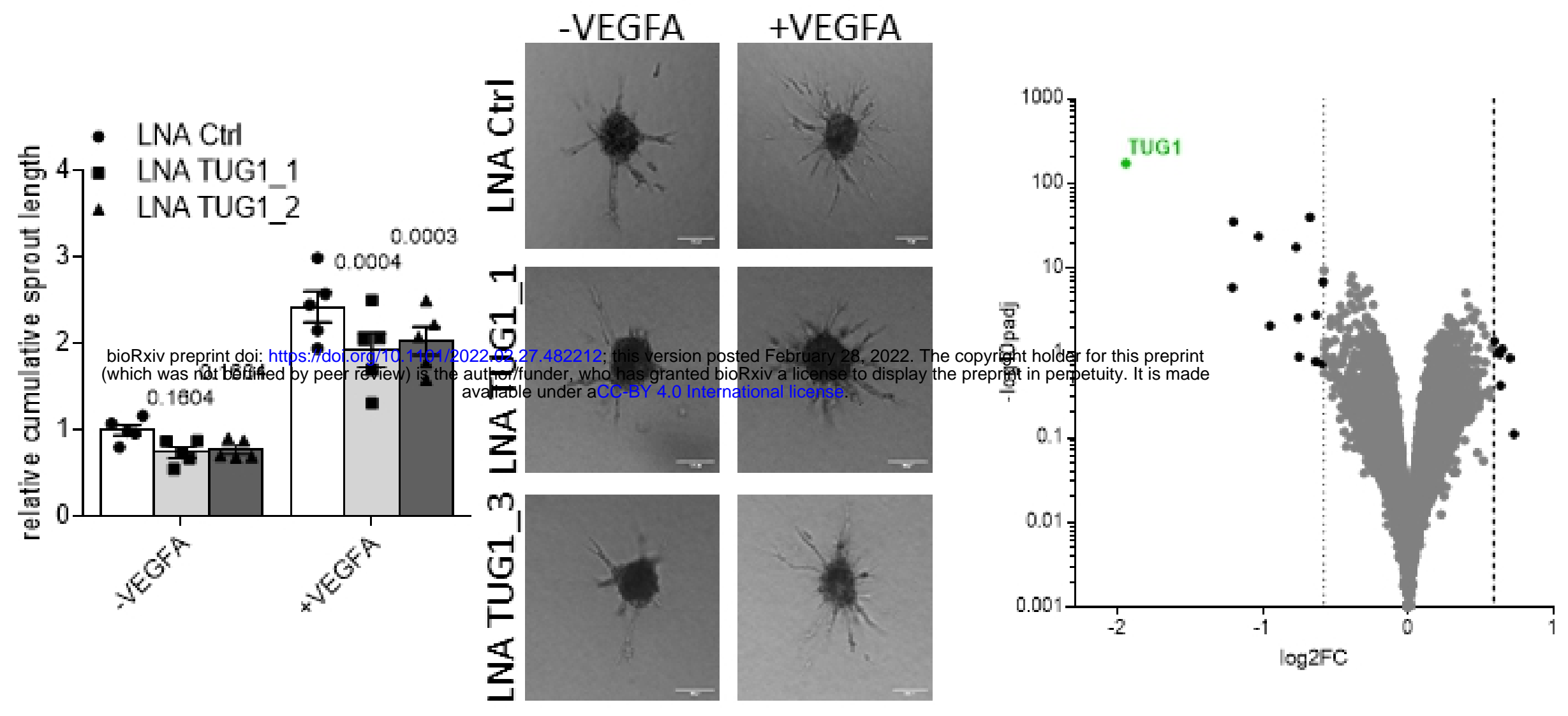

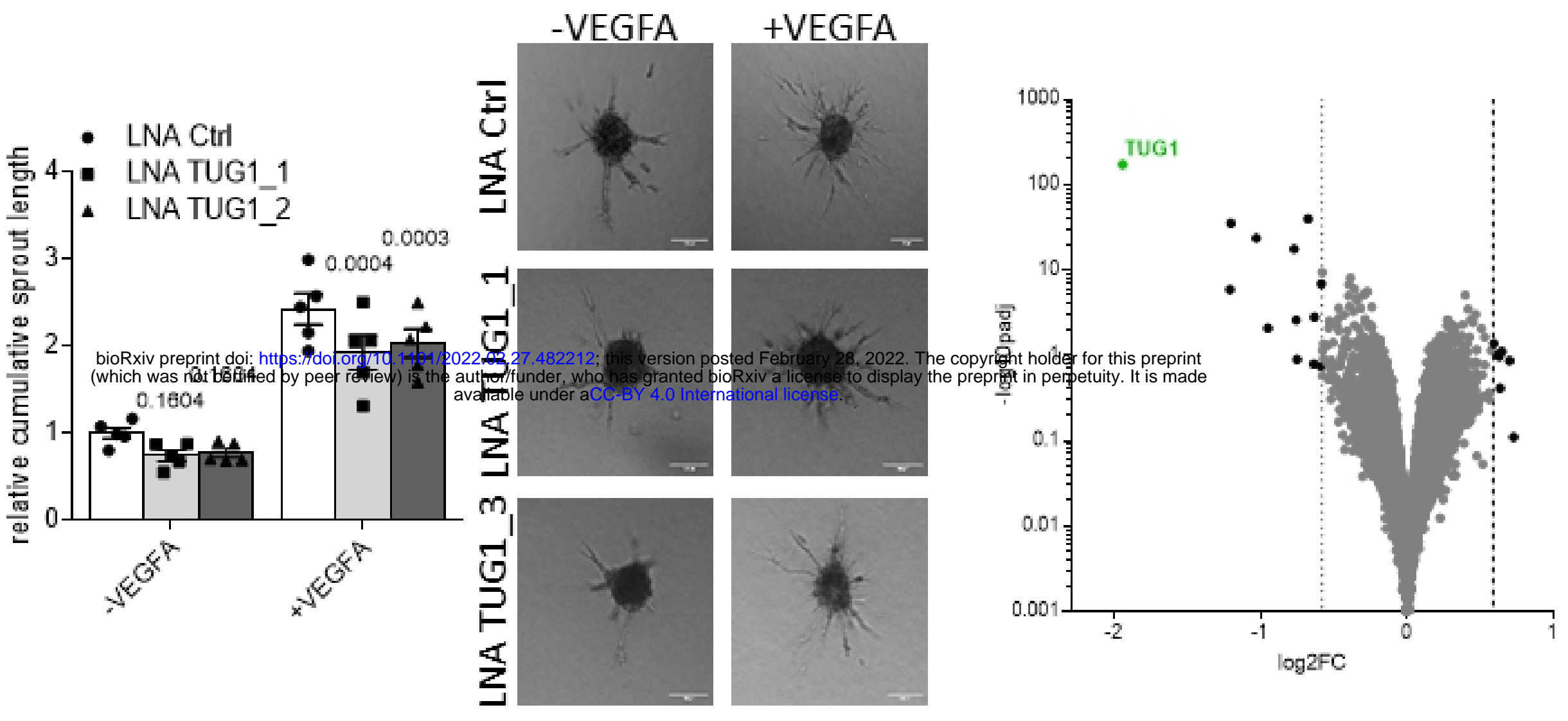

B

Bulk RNA sequencing
+VEGFA

Sprouting angiogenesis (siRNA)
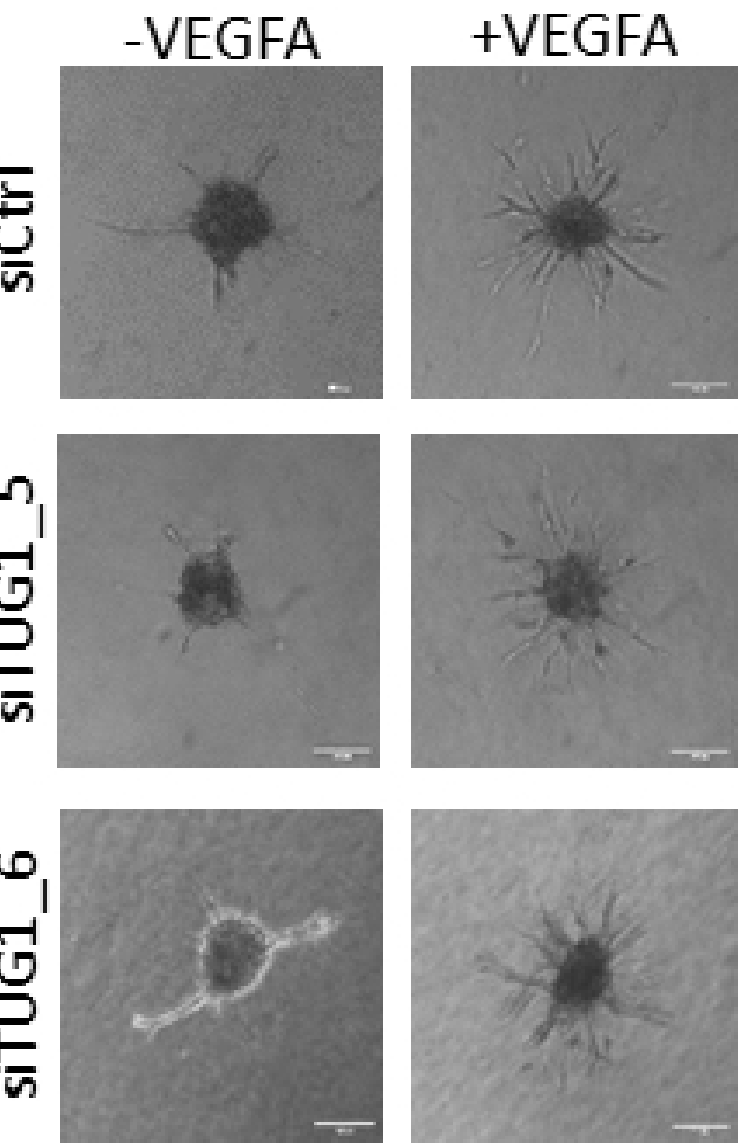